\title{
ALGEBRAIC INTEGRATION THEORY
}

\section{IRVING SEGAL}

1. Introduction. Although the theory of the abstract Lebesgue integral has strong intrinsic architectural articulation, the very importance of the theory for a variety of mathematical applications has tended to cloud its basic simplicity and elegance. The large number of approaches and formalisms, each justified by its appropriateness in connection with particular applications, has given the theory as a whole an appearance of heterogeneous complexity which is in fact significantly specious and a hindrance to a deeper understanding of the theory and to its further application. While it would probably be widely granted that integration serves as a focal point for real analysis, the focus has been more virtual than real; the ultimate convergence of the multitude of generally similar but technically distinct theories has been felt rather than proved.

On the other hand, the coherence and algebraic simplicity of the basic theory has been visible and virtually taken for granted for a long time by those working in abstract analysis. The replacement of the sigma-ring of all measurable sets by the abstract Boolean ring of measurable sets modulo null sets is natural from a fundamental statistical viewpoint, and provides probably the earliest approach displaying such features. This Boolean ring approach, which has now been elaborated by many publications, most notably those of Carathéodory and his associates, is, however, an inconvenient one for most applications, inasmuch as these deal mainly with functions, which enter into the Boolean ring approach only in a rather circumlocutory fashion. Around the later thirties there appeared, as a fruit of the spectral and representation theory originating in the well-known work of von Neumann, Stone, and Gelfand, simple abstract characterizations of measure-theoretic function spaces, as ring and/or lattices. However, no comprehensive development of integration theory along such lines took place at that time.

It was the development of a variety of new theories, rather than the desire to embellish old ones, which primarily has led to the development of a complex of results, methods, and ideas here somewhat loosely referred to as 'algebraic integration theory.' The introduction of a new term such as this requires some explanation and justifica-

The preparation of this paper was supported in part by the National Science Foundation under grant NSF G-16428 to the American Mathematical Society; received by the editors January $22,1965$. 
tion, in the light of the rapidly increasing burden which mathematicians must bear. To this end we point out that one almost universal feature of applied work is a tendency to overlook mathematical isomorphisms. In theoretical physics, for example, although analogies are frequently invoked, the concept of a rigorous isomorphism rarely occurs in a nontrivial form, although it is relevant in a number of significant ways in contemporary work. The result of this is, naturally, a tendency toward a variety of babel; each special context tends to acquire a distinctive terminology and notation, and close connections or even virtual identities between superficially distinct developments, are rendered quite obscure. This is, of course, not only uneconomical, in the duplication of labor involved, but brings a substantial linguistic encumbrance to bear on the scientific commerce between different areas and the development of interdisciplinary subjects. Even in probability theory, the most notably successful of originally applied subjects in achieving mathematical clarity and emerging in this century as a distinctive branch of mathematics, cases could be cited in which quite wrong conclusions were reached as to the relation between certain work and earlier extant theories. While the use of analogies is a partial substitute in heuristic work, it has the disadvantage of making it impossible to determine whether the results are merely plausible or actually quite correct; on occasion it even makes it difficult to determine whether the results are meaningful.

The obvious corrective for this situation is a closer adherence to a formulation of integration theory which is relatively independent of particularities and revealing of features independent of the notation. This might be called invariant integration theory, but this term might well suggest a subject quite different from this one, namely that of invariant integrals. Since the setting of the theory is naturally algebraic, in its concern with features independent of isomorphisms, the term algebraic integration theory is reasonable-although the subject is distinctly more distant from conventional algebra than is algebraic topology. Such a theory is necessarily abstract, but the term 'abstract integration theory' has already a different meaning, signifying usually the theory in which integrals are considered not necessarily over subsets of euclidean space, but over relatively general spaces, and is a more limited and quite distinct notion from that of the theory considered here, whose distinctive description as algebraic seems therefore practical.

Earliest and probably foremost among the new developments suggestive of a materially more algebraic approach to integration theory 
was the systematization of commutative spectral theory in Hilbert space. The well-known highly penetrating work of von Neumann in the thirties contained many arguments and methods having an intriguing similarity to some of those in integration theory. It was partly an interest in explicating this similarity which led to a culmination of the commutative theory in the early fifties in the determination of the structure of the general commutative ring of operators on Hilbert space, and, as a corollary, to a relatively definitive and complete form for spectral multiplicity theory in Hilbert space. It became clear that the cited similarity was not at all coincidental, but that commutative spectral theory is essentially a direct application of integration theory. The simplest, as well as a quite important, instance of this, is in the spectral resolution of a set of commuting self-adjoint operators in Hilbert space. For bounded operators this is an immediate consequence of the identification (within a transparent type of isomorphism, i.e., unitary equivalence) of a maximal abelian self-adjoint ring of operators in a Hilbert space with the ring of multiplications by bounded measurable functions (modulo null functions) on a measure space.

The substantial identity of hermitian operators in Hilbert space with real measurable functions on a measure space (with however a different notion of equivalence from that involved in the similar identification of random variables) led to the elucidation in related terms of an important part the theory of operator rings developed by von Neumann, partly in collaboration with Murray. This beautiful theory, undoubtedly one of this century's most original scientific achievements, had in a number of respects a striking but elusive similarity to various developments in integration theory. Some of this similarity, notably that between the construction of the dimension function in the work of Murray and von Neumann and the construction of group-invariant measures due to Haar, remains fundamentally elusive and may well be specious, except in the sense that Haar's discovery appears to have strongly stimulated von Neumann. On the other hand, the major part of the similarity has been thoroughly explicated by the development of a theory applicable to any operator ring on the projections of which an analogue to a measure is defined, which is basically co-extensive with the general theory of the abstract Lebesgue integral when the ring in question is commutative. The latter connection comes about precisely through the identification of hermitian operators with measurable functions; in the case of a general ring, the hermitian operators will in general not commute, and so cannot possibly be simultaneously represented by measurable 
functions, but they may nevertheless be integrated and, apart from their noncommutativity, otherwise be treated much like the measurable functions on a measure space.

The other major part of the general theory of Murray and von Neumann consists in the construction of the dimension function on the 'factors' which are in a sense complementary to the abelian rings, factors being characterized among operator rings as having trivial centers. This construction may be regarded as related to the general theory of integration in operator rings-or 'noncommutative integration,' for short-in a fashion fairly analogous to the relation between the construction of Haar measure in a locally compact group and the general theory of abstract Lebesgue integration. The simplest and most important nontrivial factor is that of all bounded linear operators on Hilbert space. The relevant noncommutative measure-or 'gage,' for short-is that which assigns to each projection the dimension of its range, or $+\infty$ if the dimension is infinite. This is countably additive, as is an abstract Lebesgue measure, and has one additional feature which compensates for the circumstance that the lattice of all projections is not Boolean, which circumstance originates in the noncommutativity of the ring; namely, this gage is unitarily invariant. These two properties are essentially the only ones required for the development of an effective integration theory parallel to and essentially inclusive of the abstract Lebesgue theory.

Naturally, instead of integrating functions, in the more general theory one integrates operators; one normally speaks, in place of the integral of a function, of the trace of an operator, in the case of the ring $B$ of all bounded linear operators, as well as for factors in general. The Lebesgue $L_{p}$ spaces have their close analogues, which are quite familiar in the case of the ring $B$, for the indices $p=1,2$, and $\infty$. The space $L_{2}(B)$ is identical with that of all so-called 'HilbertSchmidt' or, in the terminology of [102], 'finite norm' operators in the Hilbert space, with the $L_{2}$-norm identical with the HilbertSchmidt norm. The space $L_{1}(B)$ is precisely that of all operators of absolutely convergent trace, with the corresponding so-called trace norm. The space $L_{\infty}(B)$ dual to $L_{1}(B)$ turns out to be precisely the ring $B$ itself, the $L_{\infty}$ norm being identical with the usual operator bound. Simple instances of results in pure operator theory discernible from the analogy with integration theory are the forms of the continuous linear functionals on the spaces of all completely continuous operators and of all operators of absolutely convergent trace. The Riesz representation theorem for continuous linear functionals on the space of all integrable functions on a measure space is, for exam- 
ple, a special case of an analogous result valid for general rings, which specializes in the case of the ring $B$ to that indicated. Results of this type were obtained by Schatten [82] and Dixmier [15] in a purely operator-theoretic fashion, and actually the $\operatorname{ring} B$ is such a relatively simple and special one that the general integration theory does not add a great deal to what can be seen otherwise. It does, however, serve to correlate a variety of results in integration and operator theory, as well as to provide a general theory suggestive of further useful results.

On the other hand, if the interesting concrete implications of the theory were confined to conventional integration theory and to analogous results concerning the ring of all bounded operators, it would appear to be possibly expendable as an instrument for further investigation of concrete systems. One broad class of examples combining features of integration theory with the analogous theory for $B$, or for factors in general, is provided by the generalized Plancherel theory for locally compact unimodular groups. In the particularly interesting case of an open simple Lie group, this theory turns out to concern integration relative to a ring which may ultimately be identified with the direct product of the multiplication ring of a measure space with the ring $B$. The extension by Weil [113] of the classical Plancherel theorem to the case of an arbitrary locally compact abelian group proceeded from the structure theorems of Pontrjagin and van Kampen for these groups, and did not cogently display the theorem as one establishing a new species of integration on the group. The later proof by Krein of the Plancherel-Weil theorem, and especially the still later proof of Rykov [79], put this rather strongly in evidence, and at the same time freed the proof from dependence on the duality theory, which Rykov showed then followed in a natural way. Roughly speaking, what is involved in Rykov's proof is the development of a kind of integration theory for convolution operators on the group, together with the representation of the integral in a conventional way in terms of Haar measure on the dual group. More specifically, if $A$ is the operator of convolution with the function $f$ on the group, where $f$ is assumed smooth and of compact support, then the map $A \rightarrow f(e)$, where $e$ is the group unit, has a variety of nontrivial properties quite analogous to those of an integral; and it may, in fact, be represented as the integral over the dual group of the Fourier transform of $f$. This might appear as a perhaps unduly sophisticated way of looking at the theorem, were it not that the basic result is equally applicable to compact non-abelian groups, by virtue of the Peter-Weyl theorem, and is, in fact, equivalent to the theorem, if 
this is regarded as a means of expressing the $L_{2}$-norm of a function on the group in terms of the (matrix) Fourier coefficients of the function.

It was natural to surmise that for any locally compact unimodular group, there might be an analogous means of expressing the squareintegral of a function $f$ on the group in terms of the integral over an appropriate dual object of a quadratic expression in the suitably defined operator-valued Fourier transform of the function. This was indeed the substance of a formula obtained by Gelfand and Nalmark in the special case of the Lorentz group [29] by algebraically highly novel but analytically relatively classical methods. A general result of this type appeared, however, to require a theory of noncommutative integration (cf. [84]). The treatment in [112] of unitarily invariant positive linear functionals with the attainment of simple results suggested the exploration of the possible unitary invariance of the functional $f(e)$, or, rather, of some suitable modification thereof. This functional was clearly invariant under the unitary transformations corresponding to group translations, but its invariance under the much larger group of all unitary transformations in the ring of operators generated by the translations was not mathematically welldefined, since in general such a unitary transformation will carry a continuous function of compact support into one devoid of any special regularity or support properties. What was needed turned out to be a more strictly Hilbert-space than geometrical viewpoint; $f(e)$ had to be thought of as an inner product between two elements of $L_{2}$, and for the projections which play the central role in [112], the corresponding gage (of left convolution by $f$ ) had essentially to be redefined as the inner product $\left(f, f^{*}\right)$, where $f^{*}(x)=\bar{f}\left(x^{-1}\right)$, to which it is formally equal. This redefinition makes the additivity of the functional less manifest, but it is nevertheless maintained, and from this point the close connection between the von Neumann theory and the generalized Plancherel theorem was clear. The derivation of the latter in [86] along these lines was, as noted there, in considerable part applicable to general abstract algebras, as has been further substantiated by work in [88] and [101]. A less complete result, based also on the idea sketched, was given shortly afterwards in [62]; it must be noted, however, that the proof offered contains several essential gaps (cf. below).

The algebraic approach to integration theory was not at all confined to the cited examples, and, in the commutative case especially, took many forms, as indicated by the applications envisaged. The approach of Bourbaki [4A], in which the basic datum is not a mea- 
sure on a sigma-ring but a positive linear functional on the algebra of continuous functions of compact support on a locally compact topological space, led to a useful theory whose development involved a number of technical simplifications. It was particularly well adapted to the treatment of invariant measures in locally compact spaces, whose formulation as invariant integrals (i.e., linear functionals) served to circumvent difficult measurability problems which otherwise appeared and to facilitate the treatment of the existence and uniqueness of invariant measures (cf. notably [113]). Variants of this approach, which may itself be regarded as a particularly felicitous variant of developments due to Daniell [13], Banach [80], and others, have been given by many authors (McShane [64], Stone [105], to cite two readily accessible treatments), but without the local compactness of the underlying space a substantial part of the technical incisiveness is lost.

On the other hand, while this approach was quite adequate for the general treatment of integration in the geometrical types of spaces whose consideration appears to have motivated the approach, it seemed out of place in a number of other connections. In probability theory and in the mathematical theory of quantum fields, integration over infinite-dimensional spaces is involved, and the local compactness condition is out of place. To be sure, with any finite measure space, an integration-theoretically largely equivalent compact space may be associated, in fact it may be uniquely chosen so as to have the property that any bounded measurable function class, modulo the class of null functions-or, for short, bounded measurable-on the original space corresponds to a unique continuous function on the compact space. Such 'perfect' [93] or 'Stonian' [16] spaces are on occasion useful in circumventing measurability difficulties, but the apparent impossibility of describing them in explicit terms and the burden of carrying an isomorphism, rather than the identity map, throughout a problem, strongly limits their usefulness. Thus the Wiener integral, substantially over the space $C[0,1]$ of continuous functions in the interval $[0,1]$, gains nothing, so far as is visible at this point at least, through its transfer to an associated compact space, and in fact loses explicitness; the same is true in fact of most stochastic processes, which generally involve integration over some infinite-dimensional linear space.

Probability theory relates, however, to algebraic integration theory in quite another way. In a sense, the early probabilists, such as the Bernoullis, may be regarded as primitive exponents of a variety of algebraic integration theory. The random variables with which they 
worked directly were never constructed mathematically but rather treated in a highly implicit axiomatic, and in essence algebraic, fashion. In retrospect, and employing modern terminology, their basic informal axiom may be formulated as follows: the random variables form (or may be regarded as generators of) a real commutative associative algebra, on which a distinguished linear functional, the 'expectation' functional (determined frequently through the moments of generating random variables), is defined. However, thirty years ago such axioms were not quite so readily formulated, and, more significantly, if formulated would have been difficult to take seriously as a possible basis for the theory. The subsumption of the basic parts of probability theory under the theory of the abstract Lebesgue integral by Kolmogoroff in 1933 [50] relieved mathematical probabilists of a substantial burden of insecurity concerning the foundations of their subject, and in fact marked the beginning of the era of the truly mathematical development of the subject; any alternative to the fundamental idea that a random variable was simply a measurable function on a probability measure space (i.e., one of total measure unity) would have had to have the clearest kind of practicability and utility to have received any significant consideration. The mathematical viability of an algebraic approach such as that indicated was visible only at the end of the decade, following the development of the representation theory by Stone, Gelfand, and many others, while its utility did not appear until more than another decade later.

The idea that a random variable is a measurable function has therefore, despite a certain lack of physical intuitiveness, dominated probability theory in the past thirty years, and quite naturally so. While the definition in terms of an algebra-cum-linear functional may throw some light on the foundations of the subject, it is mathematically equivalent to the earlier definition, and it is only within the past decade or so that some material technical advantages for the algebraic approach have been visible. These advantages have to do with the related subjects of the general theory of analysis in Wiener space, developed principally by Cameron and Martin, and the theory of the free Bose-Einstein quantum field, which are linked through their common close connection with analysis in Hilbert space. The theory of the homogeneous chaos due to Wiener [116], for example, acquires both greater generality and simplicity through its formulation in terms of analysis in Hilbert space rather than Wiener spacea quite material change, being based on a certain duality between Wiener and Hilbert space based in turn on the Wiener stochastic 
integral, rather than on the obvious possibility of imbedding Wiener in Hilbert space. At the same time, this formulation makes it possible to bring the theory into explicit relation with relativistic quantum field theory in a way which has not been possible with Wiener space itself, which is not in any natural way a relativistic object; in these terms, for example, the Hilbert space counterpart to Wiener's chaos of degree $n$ corresponds to what, in theoretical physics, is described as the subspace (of state vectors) in which there is present at most $n$ particles. It is extraordinary, in a way, that the wave-particle duality in a Bose-Einstein field should be partly implicit in the essentially mathematically and classically motivated development of the homogeneous chaos, but this connection might not have been observed, nor could it be made so transparent, without the algebraic approach.

In the same way, the 'Fourier' transform in Wiener space studied by Cameron and Martin, which at first glance appears to have a rather limited analogy with the classical Fourier transform may be seen via the connection with Hilbert space to be a natural generalization of the Fourier-Plancherel theory from a finite-dimensional euclidean space to Hilbert space. The important question, for applications both to quantum field theory and stochastic processes of the absolute continuity of affine transformations in Wiener and related spaces, has a much simpler and more readily attainable resolution in the algebraic theory; the result may then be transformed into a corresponding one for any given stochastic process, of a more specialized nature. The theory of Gaussian integration in Hilbert space which is central here is most economically derived from an algebracum-linear functional approach in which the algebra is that of all polynomials (in the naive sense) and the expectation functional that given by the Gaussian integral.

In addition to its technical advantages, this approach serves to recapture the essential spirit of the Bernoulli approach, in its emphasis on the mathematical counterparts to the objects which from a conceptual empirical viewpoint are most directly accessible-essentially the key random variables and their moments. The axioms required for analytical effectiveness turn out to be quite few in number, as well as of immediate intuitive acceptability as descriptive of the random variables of a probability system. The origin of such axioms, in quantum and spectral theory rather than in the mathematical theory of probability itself, leads to a certain unification of the concepts of observable in quantum theory and of random variable in probability theory, while clarifying the mathematical formulations of these concepts and the relations between classical and quantum 
systems. The somewhat ad hoc and artificial character of the key phenomenological assumptions of quantum theory, to the effect that an observable is an hermitian operator in Hilbert space while a state is represented by a vector in (or essentially in) the space (cf., e.g., Dirac [14]) led von Neumann in the late twenties and earlier thirties to make a searching study of rationalizations or alternatives. This culminated in the highly original and very interesting, although basically inconclusive, study of a certain set of axioms for the observables of a quantum system as a structured variety of Jordan alge$\mathrm{bra}$, in [110]. Abandoning the attempt to treat noncommutative systems, and with a relatively more purely mathematical motivation, Stone slightly later studied conceptually simple systems abstracting commutative operator algebras, and succeeded in developing along these lines a systematic and natural approach to some of the basic parts of spectral theory in Hilbert space. Following Stone's earlier work, the theory had reached a culmination point in Gelfand's theory of normed rings; the technical effectiveness of Gelfand's emphasis on complex methods more than compensated for a certain lack of methodological purity represented by the use of such methods in a real-analytical problem, and such complex methods have played a virtually dominating role in later work on Banach algebras. At any rate, by the early forties there were available several ways of representing abstract topological-algebraic systems, descriptive of systems of random variables, simultaneously observable quantum variables, or systems of operators generated by commuting normal operators on Hilbert space, by the more familiar concrete systems of functions. In particular, random variables as implicitly defined in such work as that of the Bernoullis could be mathematically identified with the now conventional definition of a random variable as a measurable function, although the first occasion for taking note of this came a decade later, in connection with functional integration and invariant Gaussian integration in Hilbert space especially.

This subject has a somewhat curious history. An invariant integral in Hilbert space was originally sought for on a variety of purely mathematical grounds, and then abandoned as a mirage. A decade or two later a kind of symbolic functional integral was introduced in Hilbert space by theoretical physicists, who noted that it had remarkable formal possibilities for quantum field theory, but never succeeded in attaching any definite numerical value to the pseudointegrals that their formalism was concerned with (for early work of this nature, see Feynman [21], [22]). The difficulty is that there simply is no direct analogue in Hilbert space to the euclidean volume 
element in $n$ dimensions, $n<\infty$, although this is exactly the element of integration the physicists required. The way out of this difficulty is, however, indicated by one of the most familiar and fundamental results in quantum field theory, or, rather, by its proper mathematical explication. The fact that a free Bose-Einstein quantum field could be 'represented' as an assembly of harmonic oscillators was well known. A clear statement of this equivalence led directly to the formation of infinite Gaussian integrals (or infinite products of finite ones), which in an invariant treatment could only be taken over the Hilbert space representing the admissible 'single particle' states, or some closely related space identical to it at least in the case of finitedimensional systems, and invariantly attached to it. In other terms, what was involved was not an analogue to the euclidean volume element, but rather an analogue to the isotropic Gaussian distribution centered at the origin.

It was 'well known,' in an informal mathematical way, that no such object existed; nevertheless it was clear that there was mathematical substance to the equivalence between a Bose-Einstein field and a set of harmonic oscillators, and plausible that there should be an invariant formulation of this equivalence. Suggested partly by this need, and partly by the instance provided by Wiener's stochastic integral, the concept of a weak distribution arose. Given a probability distribution on a Hilbert space of sequences, say, $\left(x_{1}, x_{2}, \cdots\right)$, each coordinate $x_{i}$ becomes a well-defined random variable; conversely, if the coordinates are well-defined random variables, in the finite-dimensional case-but only in this case-there necessarily exists a probability distribution with which they are associated in the foregoing manner. The notion of weak distribution defined in this way was thus materially more general than that of a conventional probability distribution, and the isotropic centered normal distribution-or, for short, 'isonormal' distribution-fits in nicely as a weak distribution; there is an essentially unique way to assign to each vector $x$ of a given real Hilbert space a random variable $R(x)$ such that for any orthonormal set of vectors $e_{1}, e_{2}, \cdots, e_{n}$, the random variables $R\left(e_{1}\right), R\left(e_{2}\right), \cdots, R\left(e_{n}\right)$ have independent and identical Gaussian distributions centered at the origin.

It is clear that the isonormal distribution is an attribute of the Hilbert space itself, and independent of its concrete representation. The Wiener stochastic integral, in which $x(t)$ represents the WienerBrownian motion process,

$$
f(\cdot) \rightarrow \int f(t) d x(t)
$$


is a mapping from $L_{2}(0,1)$ to random variables having the defining properties of this distribution, but evidently involves much additional structure, which turns out not to be needed for a considerable part of the basic theory. But before any theory could be developed, or, in fact, the structure of the free Bose-Einstein field fully explicated, it was necessary to have a means of integrating relative to weak distributions. While an ad hoc method was available for the isonormal distribution in the light of the theoretical physical results themselves, and was construed by Friedrichs as a partial analogue to an integral in the Hilbert space, ${ }^{1}$ there was no established general method, such as was required if the Bose-Einstein field representation theorem was to be successfully related to general mathematical ideas; the abstract Lebesgue theory did not appear to apply. It was necessary to observe that the bounded measurable functionals on the Hilbert space which were dependent only on a finite number of coordinates'tame' functionals for short-formed an integration algebra relative to the integral provided by the given weak distribution for such tame functionals. In the isonormal case as well as for a fairly broad class of other weak distributions the polynomial functionals could be used in place of the bounded ones, but the algebra of tame functionals was always available and provided a simple way to connect with general integration theory.

Analysis relative to the isonormal distribution as volume element is in many ways as simple-in some ways, perhaps simpler-as analysis based on the euclidean volume element, as it has turned out, and leads to considerable mathematical illumination of the theory of free and other linear Bose-Einstein fields. It has not in itself yet led to the resolution of the problems concerning nonlinear quantum fields which in a formal way could be resolved by free use of analysis in function space, but important progress has resulted, and much light has been thrown on the origin of these problems. To mention one aspect, the nonlinear transformations in function space involved in these problems not only are not 'absolutely continuous' in the suitably generalized sense, relative to the isonormal distribution, but may well, so far as is now known, and unlike general linear transformations which may also fail to be absolutely continuous, transform the isonormal distribution into any other well-defined weak distribution at all. The weakness of a distribution involves a whole complex of significant technical differences, and while the notion

${ }^{1}$ Specifically, the representation of the eigenstates of the harmonic oscillator in a boson field as Hermite functionals (cf., e.g., von Neumann [109]) gives an inner product between such functionals, and hence between linear combinations thereof. 
has been found useful in the theory of stochastic processes (see e.g., Prohorov [76]) as well as in quantum field theory, it is far from being a panacea. In fact, the theory could possibly also be presented in terms of conventional measure theory, at the cost of considerable circumlocution, loss of manifest invariance, and, in our opinion, general transparency.

That the algebraic approach is, however, materially more than merely a convenient and invariant way to present the theory is rather strikingly indicated by the parallel consideration of the free FermiDirac quantum field. There is present an analogy between the two types of fields which, it develops, is of a remarkably far-reaching mathematical character, and is visible only from the integrationalgebra standpoint. This is because in the Fermi-Dirac case there is no invariant conventional measure space in terms of which the theory may be presented; the integration algebra in question is noncommutative, and is, in fact, the 'Clifford algebra' over a Hilbert space. This circumstance represents, incidentally, one of the very few intrusions into concrete analysis of the exceptional factors of Murray and von Neumann, inasmuch as this algebra is an approximately finite factor of type $\mathrm{II}_{1}$, in their terminology. The penetrating character of the analogy between the two types of fields is visible in a purely mathematical way from its having led in an incidental way to the construction of an analogue for the symplectic group to the spin representation for the orthogonal group ([95A], [99]), quite unsuspected by algebraists, in advance of the independent discovery of the representation, in a different context, not revealing of the analogy with the spin representation, as the basis for the understanding and extension of recent developments in number theory by Weil [114]. The utility of the concept of weak distribution is confirmed by its applicability to the noncommutative case, the 'Clifford' distribution which intervenes having many formal properties in common with the isonormal distribution, despite the lack of any formal resemblence or invariant connection whatsoever of this distribution to a conventional countably additive measure on a sigma-ring.

Thus the algebraic viewpoint in integration theory is a natural, and, in some important respects, an indispensible guide and tool in connection with a variety of applications. There are other areas where similar ideas are at first glance less clearly indicated, but where it has turned out that they provide a deeper understanding of important results. The direct integral theory of von Neumann [112], for example, originally quite involved, appears as regards some of its most applicable (but by no means all) features as a simple deduction from 
commutative spectral theory via integration-algebra methods. The fundamental theory of harmonic analysis on locally compact abelian groups develops naturally and, probably maximally, rapidly from the same line.

Representation theory for more general locally compact groups is related to these matters, the theory of so-called 'states' of operator algebras or positive definite functions on groups having many of the features of algebraic integration theory. The general lack, however, of one of the essential properties indicated earlier, namely, the unitary invariance (or, essentially, centrality, i.e., the property of the positive linear functional $E$ that $E(A B)=E(B A)$ ), is an important limitation, as a consequence of which the general integration theory for states is relatively rudimentary. For this reason it seems appropriate for the present treatment to draw its demarcation line just short of such considerations, insofar as it treats analysis on locally compact groups. Also treated only quite marginally is the general theory of rings of operators, except insofar as it is related to the more structured situations involving an integral which are our central concern.

With this brief bird's-eye view of algebraic integration theory, we turn now to specifics. Our approach will be, as already suggested, to treat the fundamental theory and to present selected illustrative material, rather than to give a compendium of all remotely relevant research with brief descriptions of each item. This means that much highly significant work must be passed over or given only marginal attention. It is believed, however, that the bibliography is sufficiently extensive to enable the interested reader to track down fairly readily the literature on matters of special concern to him which relate materially to the present subject. We apologize in advance both to those who might prefer more of a compendium arrangement and to those who might prefer to have a completely general account uninterrupted by technical asides and illustrations-as well as to the authors of important papers which have been overlooked or considered to be insufficiently relevant or illustrative to describe or mention.

2. Integration algebras. The simplest prototypical case, as well as one which can be considered theoretically basic, is that of the algebraic characterization of a finite measure space. As indicated, this is readily done in terms which are both mathematically useful and probabilistically intuitive.

Theorem. Let $(A, E)$ be a system composed of a real commutative associative algebra $A$ with unit, and a linear functional $E$ on $A$, with the properties 
(i) $E\left(A^{2}\right) \geqq 0$, and $=0$ if and only if $A=0 \quad(A \in A)$,

(ii) for any element $B$ of $A$ there exists a constant $b$ such that

for all $A$ in $A$.

$$
E\left(A^{2} B\right) \leqq b E\left(A^{2}\right)
$$

Then $(A, E)$ is isomorphic to a dense subalgebra of $L_{\infty}(M)$ for some finite measure space $M$, with the integral as linear functional.

Here $L_{\infty}(M)$ denotes the algebra of all equivalence classes of bounded measurable functions on $M$ - two functions being defined as equivalent if they agree except on a null set. 'Finite measure space' means, as usual, the system consisting of a set $S$, a distinguished Boolean sigma-ring $S$ of subsets of $S$ which includes $S$ itself, and a real non-negative countably additive function $s$ on $S$. 'Dense' may be defined in various ways, the simplest, perhaps, being the usual topological density relative to that induced on $L_{\infty}(M)$ from the $w^{*}$ topology on the dual of the space $L_{1}(M)$ via the well-known isomorphism of the dual with $L_{\infty}(M)$; it would be quite equivalent to say that the subalgebra is dense in $L_{2}(M)$ in the $L_{2}$-metric, or, alternatively, that it is determining, in the sense that the least sigma-ring with respect to which every element of the subalgebra is measurable includes every measurable set, within a null set. Note that, conversely, it is essentially obvious that any subalgebra of $L_{\infty}(M)$, with the integral as linear functional, does indeed satisfy (i) and (ii). Note also that if $E(I)=1$, where $I$ denotes the unit, then the postulated conditions on $E$ are physically plausible ones for the bounded random variables of a probabilistic system, in the light of a moment's reflection.

The proof of the Theorem is not at all complicated, but involves in an essential way two relatively recent, if now widely known, mathematical developments: (a) the association with a positive linear functional on an algebra of a representation of the algebra by operators in a Hilbert space; (b) the elementary theory of Banach algebras. With the inner product

$$
(A, B)=E(B A),
$$

the algebra $\boldsymbol{A}$ acquires also the structure of an incomplete Hilbert space whose completion will be denoted as $H$. If, for any element $A$ of $A, \phi_{0}(A)$ denotes the operator on $A, B \rightarrow A B$, then by (ii) $\phi_{0}(A)$ is a bounded linear operator on $A$, and so extends uniquely by continuity to a bounded linear operator $\phi(A)$ on all of $H$. The map $A \rightarrow \phi(A)$ is an algebraic isomorphism, and $E$ may be expressed in the form $E(A)=(\phi(A) I, I)$, by virtue of which observations it is no essential loss of generality to assume that $A$ is a commutative algebra 
of self-adjoint operators on a real Hilbert space $H$, containing the identity operator $I$, and $E$ has the form $E(A)=(A z, z)$ for some vector $z$ in $H$ whose transforms $A z$ under $A$ form a dense subset of $H$.

If $A$ and $B$ are bounded self-adjoint operators on a Hilbert space, it is readily verified that $\left\|A^{2}\right\|=\|A\|^{2}$ and $\left\|A^{2}-B^{2}\right\| \leqq \max \left(\left\|A^{2}\right\|\right.$, $\left.\left\|B^{2}\right\|\right)$. The general theory of Banach algebras asserts that the closure of $\boldsymbol{A}$ is naturally homomorphic to a subalgebra of the algebra of all complex-valued continuous functions on a certain compact Hausdorff space, the 'spectrum' of $\bar{A}$ (cf., e.g., [83]). The first property of the norm shows that this homomorphism is, in fact, an isomorphism, in which the operator bound and the function supremum correspond. The second property serves to exclude the case of complex-valued functions - as would substantially equally well any of a wide variety of other conditions-i.e., the image algebra consists exclusively of real functions. The Stone-Weierstrass theorem then implies that the image consists of all real-valued continuous functions on the spectrum $\Gamma$. Now $E$ extends in an obvious way to $\bar{A}$, and so corresponds in the isomorphism to a positive linear functional on the real-valued continuous functions on $\Gamma$. This in turn, by the generalized Riesz theorem, is the integral relative to a finite measure on the Borel subsets of $\Gamma$, and the required measure space has been obtained.

It is natural to raise the question of the uniqueness of the associated measure space. Indeed it is unique, within so-called weak equivalence [93], a simple concept but one not needed for most applications of the Theorem. In fact at this point we may postpone further consideration of algebraic integration theory and turn to some illustrative applications.

Although probably surprising to any one not familiar with the theory of Banach algebras, the Theorem has a rather innocuous if reasonably pleasant appearance. It has, however, fairly direct consequences of considerable substance. Most important among these is the subsumption of commutative spectral theory in Hilbert space under integration theory. This subsumption may conveniently be based on

COROLlaRy 1. A maximal abelian self-adjoint algebra of bounded linear operators on a complex Hilbert space is unitarily equivalent to the multiplication algebra-i.e., the algebra of all muliplications by bounded measurable functions, acting on $L_{2}-$ of a measure space.

This is in a way a definitive formulation for the simultaneous spectral resolution of commuting self-adjoint operators in Hilbert space, 
and a quite natural one in the context of the other major realanalytical theory developed around the same time, that of Lebesgue. The proper treatment of the continuous spectrum which Hilbert discovered is seen to be virtually equivalent to the proper treatment of integrals, i.e., continuous sums, which Lebesgue developed. Since any commuting set of bounded self-adjoint operators on a Hilbert space may be imbedded in a maximal such algebra, Corollary 1 implies that for all Hilbert space purposes, the most general collection of such operators is simply a set of multiplications by real bounded measurable functions acting on $L_{2}$ over a measure space. The operators are thereby simultaneously 'diagonalized' in a quite explicit sense, a materially stronger result than that provided by the familiar formalism of Hilbert involving integrals relative to spectral families (resolutions of the identity).

For the proof, consider first the case in which there exists a cyclic vector $\boldsymbol{z}$ for the ring $\boldsymbol{A}$, i.e., one such that $\boldsymbol{A z}$ is dense in the Hilbert space $H$. With the definition $E(A)=(A z, z)$, the system $(A, E)$ is an integration algebra, satisfying the conditions of the Theorem, so that there exists a measure space $M$ and an isomorphism $\phi$ of $\boldsymbol{A}$ into a dense subalgebra of $L_{\infty}(M)$ such that $(A z, z)=\int_{M} \phi(A)$. It follows that the map $A z \rightarrow \phi(A)$ is a well-defined isometry of a dense subset of $H$ onto a dense subset of $L_{2}(M)$, and so extends uniquely to a unitary transformation $U$ of $H$ onto $L_{2}(M)$. It is straightforward to check that $U A U^{-1}$ is the operation of multiplication by $\phi(A)$. Since the multiplication algebra of $M$ is abelian and since $U A U^{-1}$ must be maximal abelian, as $\boldsymbol{A}$ is, $U \boldsymbol{A} U^{-1}$ must be all of the multiplication algebra, which completes the proof for the case in which there exists a cyclic vector.

The key observation in the reduction of the general case to that in which there exists a cyclic vector is that if $z$ is any vector in $\boldsymbol{H}$, then the set $\boldsymbol{A}_{\boldsymbol{M}}$ of all restrictions of elements of $A$ to the closure $\boldsymbol{M}$ of $\boldsymbol{A z}$ is again maximal abelian - and, in addition, possesses the cyclic vector 8 . By transfinite induction, $H$ is a direct sum $\sum_{\lambda} H_{\lambda}$ of subspaces of this type. Each restriction algebra $A \mid H_{\lambda}$ is maximal abelian and has a cyclic vector, so that it is equivalent to the multiplication algebra of a measure space $M_{\lambda}$. It is basically quite straightforward to show that $\boldsymbol{A}$, as the direct sum of the restriction algebras, is equivalent to the multiplication algebra of the direct sum of the measure spaces $M_{\lambda}$, when the latter notion is defined in the obvious way.

The deduction of the conventional form of the spectral theorem for commuting normal operators is fairly well illustrated through the consideration of the case of a single self-adjoint operator. 
Corollary 2. For any bounded self-adjoint operator $A$ on a Hilbert space $H$, there exists a unique countably additive function $E$ from the Borel subsets of the reals to the projections on $H$ such that

$$
A=\int \lambda d E(\lambda),
$$

in the sense that, for all vectors $x$ and $y$ in $H,(A x, y)=\int \lambda d m_{x, y}$, where $m_{x, y}(B)=(E(B) x, y)$ for all Borel sets $B$. Furthermore, $E$ vanishes on sets disjoint from the interval $[-\|A\|,\|A\|]$.

For the proof, assume $A$ is the operation of multiplication by the real measurable function $A$ on the measure space $M$, and define $E(B)$ for any Borel set $B$ as the operation of multiplication by the characteristic function of $a^{-1}(x)$. The countable additivity of $E$, etc., follows straightforwardly from elementary Lebesgue integration theory. This shows the existence; the uniqueness follows in a straightforward and familiar manner through the development of a theory of integrals of the type $\int f(\lambda) d E(\lambda)$. This, at the same time, serves to show the uniqueness of the notion of the Baire function $f(A)$ defined as the operation of multiplication by $f(a(x))$.

Many other familiar results are readily derived from Corollary 1, of which only a few will be cited. The structure of a compact (or completely continuous) self-adjoint operator, i.e., transforming bounded into relatively compact sets, is readily deduced from elementary integration theory, which shows that its proper vectors $x$, i.e., vectors such that $A x=\lambda x$ for some scalar $\lambda$, the associated proper value, span the Hilbert space, which, in fact, has a maximal orthonormal set consisting of proper vectors, and that there exist only finitely many elements of such a set for which the associated proper values exceed any given positive number. The Peter-Weyl theorem, to the effect that, for any compact group $G$, the Hilbert space $L_{2}(G)$ (relative to Haar measure) is the direct sum of finite-dimensional subspaces each of which is invariant and irreducible under the operations $f(x) \rightarrow f(a x)$ for all $a$ in $G$, is readily deduced, in the following manner: If the direct sum $K$ of a maximal collection of mutually orthogonal such subspaces is not all of $L_{2}(G)$, choose an element $f \neq 0$ which is orthogonal to $K$, let $g$ denote the convolution of $f$ with its adjoint $f^{*}$, where $f^{*}(x)=\bar{f}\left(x^{-1}\right)$, and consider the operation $A$ on $L_{2}(G)$ of right convolution by $g$. It is easily seen that $A$ is distinct from zero, and commutes with all left translations; using the compactness of $G$, it follows without difficulty that $A$ is a compact operator. Left translations therefore leave invariant the finite-dimensional proper space 
belonging to any nonzero proper value of $A$, contradicting the maximality assumption. A simple deduction from the Peter-Weyl theorem is the following classical generalization: Any continuous unitary representation of a compact group is a direct sum of finite-dimensional irreducible such representations.

Somewhat more complicated than the foregoing deductions is that of the well-known theorem of Stone which can be regarded as the analogue of the last result cited for the additive group of the real numbers. Instead of discrete sums, continuous ones, i.e., integrals, are involved. The relative strength of the full diagonalization result (Corollary 1) over the older formulation in terms of spectral measures, as well as over the highly algebraic results which do not provide a unitary equivalence but only an algebraic isomorphism with a ring of continuous functions, is illustrated by the simplicity of the deduction of this fundamental result. The proof serves also to illustrate the utility of perfect spaces in circumventing measurability difficulties. A finite perfect space may be defined as a pair $(\Gamma, m)$ consisting of a compact space $\Gamma$ and a regular (or 'Radon') measure $m$ on the Borel subsets of $\Gamma$ having the property that, for any bounded measurable function, there is a unique continuous function differing from it only on a null set. The proof of Corollary 1 establishes, incidentally, the somewhat surprising, and occasionally quite useful,

Lemma. If the maximal abelian algebra in Corollary 1 has a cyclic vector, the corresponding measure space may be chosen so as to be perfect.

Corollary 3. Any continuous one-parameter unitary group on a complex Hilbert space is unitarily equivalent to a group of multiplication operators of the form

$$
U(t): f \rightarrow e^{i k t} f \quad\left(f \in L_{2}(M)\right)
$$

for some measure space $M$ and real measurable function $k$.

It is sufficient to consider the case in which there exists a cyclic vector for the given one-parameter group, say $U(t),-\infty<t<\infty$, for the entire space is the direct sum of invariant subspaces in each of which a cyclic vector exists, and the desired function $k(x)$ for the entire space is merely a common extension of the functions associated with each constituent space, as in the proof of Corollary 1 . Now let $U(t)$ be the operation of multiplication by the function $u_{t}(\lambda)$ on the perfect space $\Lambda$, which function may be assumed to be continuous on $\Lambda$ for each $t$. Since $U(t) U\left(t^{\prime}\right)=U\left(t+t^{\prime}\right)$ for all real $t$ and $t^{\prime}, u_{t}(\lambda) u_{t^{\prime}}(\lambda)$ and $u_{t+t}(\lambda)$ differ only on a null set, and, being continuous, are 
identical. It follows that the function $\chi_{\lambda}$ on the reals defined by the equation,

$$
\chi_{\lambda}(t)=u_{t}(\lambda),
$$

is a character of the additive group of the reals; if this character is continuous it is of the form $\chi_{\lambda}(t)=e^{i k(\lambda) t}$ for some real $k(\lambda)$. It suffices now to show that $\chi_{\lambda}$ is continuous for almost all $\lambda$, since the measurability of $k$ then follows from the observation that

$$
k(\lambda)=\lim _{n \rightarrow \infty} n\left(u_{1 / n}(\lambda)-1\right)
$$

outside the exceptional null set.

This follows by a familiar smoothing method. Specifically, let $f$ be any Lebesgue integrable function on the reals, set $W_{f}=\int U(t) f(t) d t$ (where the integral may be defined weakly), and note that $U(s) W_{f}$ $=W_{f_{s}}$, where $f_{s}(t)=f(t-s)$. It results that, for all $\lambda$,

$$
\chi_{\lambda}(s) r_{0}(\lambda)=r_{s}(\lambda) \text {, }
$$

where $r_{s}$ is the continuous function on $\Lambda$ corresponding to $W_{f_{t}}-$ which operators all commute with each other and the $U(s)$, so that all may be simultaneously diagonalized. A simple estimate shows that

$$
\begin{aligned}
\left|r_{s}(\lambda)-r_{0}(\lambda)\right| & \leqq\left\|\int(t) f_{s}(t) d t-\int U(t) f(t) d t\right\| \\
& \leqq \int|f(t+s)-f(t)| d t \rightarrow 0
\end{aligned}
$$

as $s \rightarrow 0$, showing that $\chi_{\lambda}(s) r_{0}(\lambda)$ is a continuous function of $s$ at $s=0$, or that $\chi_{\lambda}(s)$ is similarly continuous for all $\lambda$ such that $r_{0}(\lambda) \neq 0$; being a character, it is then continuous everywhere. It now suffices to show that the closed set $N$ of $\lambda$ 's such that $r_{0}(\lambda)=0$ for all $f$ is a null set. If a sequence of $f$ 's is chosen which converges to the Dirac delta function, the corresponding $W_{f}$ 's will converge strongly to the identity operator, and the corresponding functions $r_{0}$ to the function which is identically one, in a sense which precludes their common vanishing except on a null set.

Stone's theorem naturally suggests two further developments: (i) the determination of the structure of the general continuous unitary representation of an arbitrary locally compact abelian group; (ii) the diagonalization of unbounded self-adjoint operators, such as are involved in the formulation of the theorem as the assertion that $U(t)$ $=e^{i t \boldsymbol{B}}$ for some self-adjoint operator $H$. The proof of Corollary 1 applies essentially without change to the generalized situation in 
which the additive group of the reals is replaced by a general locally compact group. An additional point must, however, be considered in the general case in order to deduce the existence of a corresponding spectral resolution based on the character group, rather than on some measure space-i.e., to deduce the result first obtained by M. A. Nalmark:

Corollary 4. Any continuous unitary representation $U$ of a locally compact abelian group $G$ on a complex Hilbert space $H$ has the form

$$
U(a)=\int_{G^{*}} \chi(a) d E(\chi)
$$

for some projection-valued measure on the character group $G^{*}$.

The character group $G^{*}$ forms a locally compact topological group relative to the compact-open topology here envisaged. The projection-valued measure $E$ may be taken as defined on either the Baire or the Borel subsets of $G^{*}$, and, as before, is countably additive. For the proof the real numbers $s$ and $t$ occurring in the preceding proof are replaced by elements of $G$. Now $r_{a}(\lambda)$ is continuous not only as a function of $\lambda$ for fixed $a \in G$, but is a uniformly continuous function of $a \in G$ for fixed $\lambda$, by the estimate given earlier. It is, therefore, a jointly continuous function of $a$ and $\lambda$, and it follows by a familiar argument that on the complement of the exceptional null set $N$, the map $\lambda \rightarrow \chi_{\lambda}$ from $\Lambda$ into $G^{*}$ is continuous; $E\left(B^{*}\right)$ may then be defined as the operation of multiplication by the characteristic function of the inverse image of $B^{*}$ under this map from $\Lambda-\mathrm{N}$ into $G^{*}$, and satisfies the indicated equation by elementary integration theory, as earlier.

The treatment of unbounded self-adjoints is essentially trivially reducible to that of the bounded case through the use of the Cayley transform, i.e., in a simpler fashion than in the treatments employing the older formulation in terms of spectral measures.

Corollary 5. $A$ (partially defined) operator $A$ in a Hilbert space is unitarily equivalent to the operation of multiplication by a real measurable function on $L_{2}$ over a measure space (with the domain of all squareintegrable functions for which the resulting function is again in $L_{2}$ ) if and only if $A=A^{*}$.

The "only if" part is a matter of elementary integration theory; we indicate therefore only the "if" part.

The unitary Cayley transform $U=(A+i I)(A-i I)^{-1}$ may be assumed to be the operation of multiplication by a function of absolute 
value one, which can attain the value one only on a null set, since $U$ can leave no vector except zero strictly invariant. The inverse Cayley transform $i(U+I)(U-I)^{-1}$ therefore exists and is a multiplication operator which is readily identified with $A$.

There is one further result concerning maximal abelian algebras which is essential in multiplicity theory as well as generally useful.2

ScholiUm. Two maximal abelian self-adjoint algebras of operators on Hilbert space which are algebraically isomorphic-or whose Boolean rings of projections are such-are unitarily equivalent.

A purely Hilbert-space proof may be given, but the original integration-theoretic proof is more illuminating. It may be assumed that the algebras in question are the multiplication algebras of measure spaces $M$ and $M^{\prime}$, each of which is a direct sum of finite measure spaces. The isomorphism $\phi$ of $L_{\infty}(M)$ onto $L_{\infty}\left(M^{\prime}\right)$ carries the measure $m$ on $M$ into a measure $m_{1}$ on $M^{\prime}$ which is mutually absolutely continuous with the given measure $m^{\prime}$ on $M^{\prime}$, and the mapping

$$
f \rightarrow \phi(f)\left(d m_{1} / d m^{\prime}\right)^{(1 / 2)}
$$

is then an isometry of a dense submanifold of $L_{2}(M)$ onto a dense submanifold of $L_{2}\left(M^{\prime}\right)$ whose unique unitary extension may be seen to implement the stated unitary equivalence.

There is a delicate point in this argument in the applicability of the Radon-Nikodym theorem, whose statement in textbooks is usually restricted to sigma-finite spaces. It is, however, not difficult to show that for any direct sum of finite measure spaces (not necessarily countable) the theorem is applicable, provided that any set of finite measure relative to one measure is at most a countable union of sets of finite measure relative to the other, apart from a null set. This condition is satisfied here. In the case of an algebraic isomorphism between corresponding Boolean rings, the same proof, slightly modified so as to apply to simple functions rather than arbitrary bounded and square-integrable ones, is effective.

The reduction theory here presented for abelian algebras can be extended to more general algebras to some extent, and provides a simplified approach to the reduction theory of von Neumann [112]. In $[87, \mathrm{I}]$ and slightly later in [36] the reduction of the Hilbert space as a direct integral of subspaces is carried out as a corollary to the

2 This Scholium and the earlier Corollary 1 form the major part of the theory not contained in the work of H. Nakano and F. Wecken on multiplicity theory in Hilbert space which was needed for the development of the general structure theory for abelian rings given in $[87,1]$. 
simpler and analytically more convenient decomposition of a positive linear functional as an integral of more elementary functionals, via the Radon-Nikodym theorem. The same approach clarifies the theory of eigenfunction expansions for differential and other suitable operators, as pointed out by Mautner [63], and developed in later research in the theory of partial differential equations.

3. Abelian harmonic analysis on locally compact groups. There is no very sharp line between the theory of group representations and of general harmonic analysis. However, the former is mainly concerned with the determination and study of the irreducible or other elementary representations of the group, while the latter has to do with functions and function spaces on the group or homogeneous spaces thereof, and, especially, with the decomposition and transformation of function spaces in such manners as to decompose a given function space into elementary constituents or to provide an explicit diagonalization or analogue thereof for given operators of interest in these function spaces. Roughly speaking, harmonic analysis usually involves qualitatively more structure than the theory of group representations per se, the additional structure being basically of an integration-theoretic character. For this reason it is appropriately considered here, without undue overlapping with the recent exhaustive article of Mackey entitled Infinite-dimensional group representations, Bull. Amer. Math. Soc. 69 (1963), 628-686.

Again, there is no very sharp distinction between abelian and nonabelian aspects of harmonic analysis. Even in the case of abelian groups, some noncommutativity is involved, that of group translations and of multiplication operators, for example, while in the case of quite non-abelian, e.g., simple Lie groups, an abelian problem such as the diagonalization of a given set of commuting operators may be rather fundamental. As used here, 'abelian' is a descriptive word indicating that an abelian set of operators plays an important part, which means in practice that the theory of abelian systems in Hilbert space is relevant; it is intended as a convenient term for analytical purposes, rather than as one indicative of a fundamental distinction.

There is another distinction which should perhaps be mentioned before the relations with integration theory are gone into, namely that between the $L_{1}$ - and $L_{2}$-theories. The former theory is a relatively highly refined one, some of whose key questions are still unresolved - and quite conceivably incapable of simple resolution-in the simplest case of analysis on the line. In contrast, the $L_{2}$-theory has made substantial progress in the past two decades in the case of 
fairly general groups, including open simple Lie groups, and has significant relationships with a number of broad scientific areas, including quantum mechanics, number theory, and the theory of group representations already cited; it is, however, a relatively simple theory in the case of the real line, having been basically complete by the middle thirties. Apart from the obvious connection of an $L_{1-}$ space with integration, the $L_{1}$-theory is not related, so far as is now visible, to integration theory, being rather associated with the theory of Banach algebras. For these reasons and also because only the most rudimentary aspects of the $L_{1}$-theory are relevant to the $L_{2}$-theory, only the latter will be discussed here. The general $L_{p}$-theory is more closely related to the $L_{2}$-theory, but for reasons of brevity it also will not be considered here.

The crucial $L_{2}$-theorems in the case of the real line (or, more generally, a real vector group) have frequently been considered to be those of Plancherel [74] and of Stone [102A] or Bochner [2A], which latter result is from a contemporary viewpoint essentially equivalent to that of Stone. There is, however, a third theorem which is naturally bracketed with these; it has escaped general attention until recently because its primary applications have been to quantum mechanics. This is the theorem of von Neumann [107] on the uniqueness of the Schrödinger representation for the Heisenberg commutation relations. In the later thirties, the theorems of Plancherel and Bochner were extended to general locally compact abelian groups by Weil, who basically (and ingeniously) combined classical methods with strong use of the Pontrjagin-van Kampen structure theorem for such groups. The later proofs of the Plancherel theorem by Kreln and Rykov were independent of this theory, and, in fact, provided an alternative and economical approach to the Pontrjagin-van Kampen duality theory. At the same time, these proofs may be regarded as indicative of a basically integration-theoretic character to the generalized Plancherel theorem. Around a decade later, von Neumann's result was extended to separable locally compact abelian groups by Mackey [55], employing an altogether different method which made strong use of results of and ideas related to abelian operator ring theory. Slightly later, Loomis [54] gave a proof which avoided the separability hypothesis and was technically more elementary, but relatively intricate, requiring in fact the pieceing together of locally defined equivalences, in a manner surely possible but tiresome to carry out in detail ; and indeed it is not carried out in the cited paper. The result could be formulated and proved in a basically similar manner for non-abelian groups as well. 
The von Neumann theorem and its generalization have now been seen to play a role not only in quantum mechanics, but also in number theory, prediction and scattering theory; at the same time, they are basically more powerful than the Plancherel theorem and its generalization. Neither the Mackey proof nor the Loomis proof makes use of the latter or of duality theory, while, on the other hand, the $L_{2}$-Fourier transform may be defined through the use of the generalized von Neumann theorem. It requires only the identification of the transform as thus defined with that given by the usual integral formula as in the Plancherel theorem, therefore, to subsume the Plancherel and duality theorems under the generalized von Neumann theorem, and a simple means to establish this identification has been given by Nakamura and Umegaki [69]. Together with the following simple deduction of the basic case of the generalized von Neumann theorem from algebraic integration theory, these notions provide a most economical approach to the $L_{\mathbf{2}}$-theory on a locally compact abelian group, which emerges in a natural way as a corollary to the basic results and ideas of the preceding section.

Scholium. Let $G$ be a locally compact topological group, let $U$ be a continuous unitary representation of $G$ on a Hilbert space $H$, and let $\phi$ be a homomorphism of the algebra $\boldsymbol{A}$ of all continuous functions of compact support on $G$ into the continuous linear operators on $H$, taking real functions into self-adjoint operators, and continuous relative to the uniform topologies. Suppose that

$$
U(a) \phi(f) U(a)^{-1}=\phi\left(f_{a}\right), \text { where } f_{a}(x)=f\left(a^{-1} x\right),
$$

for all $a$ in $G$ and $f$ in $A$, and that, in addition, $\phi$ has simple spectrum (i.e., its range generates a maximal abelian algebra). Then the system $(U, \phi, H)$ is unitarily equivalent to the system $\left(U_{0}, \phi_{0}, L_{2}(G)\right)$, with

$$
U_{0}(a): h(x) \rightarrow h\left(a^{-1} x\right), \quad \phi_{0}(f) h(x)=f(x) h(x) \quad\left(h \in L_{2}(G)\right) .
$$

There is no essential difference between a homomorphism such as $\phi$ and a spectral measure on the Baire subsets of $G$, and in the statements given by Mackey and Loomis the latter formulation is employed. For any spectral measure $E$ on the Baire subsets of a locally compact Hausdorff space $X$ (assumed as always to be countably additive), there is a homomorphism $\phi$ defined by the equation $\phi(f)$ $=\int f d E$, and, conversely, every $\phi$ with the indicated properties has this form for a unique spectral measure $E$. One of the key lemmas is perhaps more illuminatingly stated in terms of a spectral measure. In this connection a spectral measure $E$ on a space $X$ will be said to be 
quasi-invariant under a continuous group $S$ of invertible measurable transformations if for every transformation $t \in S$ there exists a unitary transformation $W(t)$ such that

$$
E(t(B))=W(t) E(B) W(t)^{-1}
$$

for all Baire sets $B$ in $X$, and if, in addition, the mapping $t \rightarrow W(t)$ is a continuous representation of $S$.

Lemma. A spectral measure on a locally compact group $G$ which is quasi-invariant under $G$ is absolutely continuous relative to Haar measure.

That is, for any Baire null set $\mathrm{N}$ in $G, E(\mathrm{~N})=0$, where $E$ is the spectral measure in question. A result of this general nature appears first for the case of the additive group of the reals in the work of Plessner [75] and for more general groups in Mackey [55], who deals with ordinary measures on separable groups. Although the following proof is brief, the lemma represents one of the two crucial analytical points in the proof in the present and related scholiums, apart from general integration and Hilbert-space theory, and from a broader viewpoint may be regarded as the analogue for spectral measures of the uniqueness of Haar measure (which plays roughly a comparable role in Loomis' proof).

First note that it suffices to consider the case in which $G$ is a countable union of compact subsets, for any group $G$ has an open and closed subgroup of this character, say $G_{0}$, and the result for the sigmacompact case would imply absolute continuity of the restriction to $G_{0}$ as well as the restriction to each translate of $G_{0}$, which yields the more comprehensive result stated in the Lemma inasmuch as every Baire set is a countable union of sets contained in such translates. Note next that it suffices to consider the subcase in which there exists a countable set of vectors $x_{i}$ in $H$ such that if $P$ is any projection in the image of $E$ which annihilates all the $x_{i}$, then $P=0$. For if $\mathrm{N}$ is a null set for which $E(\mathrm{~N}) \neq 0$, then $E(\mathrm{~N}) x \neq 0$ for some $x$, say $x_{1}$. Now $G$ is the union of compact subsets $C_{n}$, and the $W\left(C_{n}\right) x_{1}$ are compact and hence separable subsets of $H$. If now $x_{1}, x_{2}, \ldots$ is a sequence of elements in these subsets which are dense in their union, the closed linear subspace $K$ spanned by the $P x_{i}$ as $P$ ranges over the range of $E$ and $i=1,2, \cdots$ is invariant both under the $P$ and the $W(a)$. Now setting $E^{\prime}(B)=E(B) \mid K$ and $W^{\prime}(a)=W(a) \mid K, E^{\prime}$ is again a quasi-invariant spectral measure on $G$, whose representation space contains a countable set of the stated type. If the result is established in this case, it therefore follows that $E^{\prime}=0$ and hence that $E(B) x=0$, 
a contradiction, completing the proof of the reduction to the indicated subcase.

To deal with the subcase it suffices in turn to show that if $B$ is a Baire set of positive Haar measure, then either $E(B) \neq 0$ or else $E$ vanishes identically. For if this has been established and if $\mathrm{N}$ is any null set such that $E(\mathrm{~N}) \neq 0$, set $Q$ for the supremum of the $W(a) E(\mathrm{~N}) W(a)=E(a \mathrm{~N})$ as $a$ varies over $G$; since a countable subset of $G$ may be chosen for which these projections have the same union, $Q$ has the form $E\left(N^{\prime}\right)$ for some null set $N^{\prime}$. The new quasi-invariant spectral measure $E^{\prime}(B)=Q E(B)$ therefore vanishes on the complement of the null set $N^{\prime}$, and hence on some Baire set $K$ of finite positive Haar measure, as well as on all its left translates. Appealing now to the fact that the convolution of the characteristic functions of two such sets $K$ is continuous, and so bounds a positive multiple of the characteristic function of some nonempty open set, it follows, on integrating the relations $E(a K)=0$, with respect to $a$ over the other set that $E$ vanishes on some nonempty open set, which, by virtue of its quasi-invariance, implies its identical vanishing.

The other key analytical point in the proof has to do with the measurability aspects of the 'multiplier' and multiplier relations which intervene. It follows from the foregoing that the map $E$ is an algebraic isomorphism from the Boolean ring of Baire subsets of $G$ modulo null sets into a ring of projections on $H$ of simple spectrum, so that there exists a unitary equivalence of $H$ with $L_{2}(G)$ carrying the $E$ 's and the $\phi$ 's into simple multiplication operations. It is, therefore, no essential loss of generality to suppose that $H=L_{2}(G)$ and $\phi=\phi_{0}$. Setting $W(a)=U_{0}(a)^{-1} U(a), W(a)$ commutes with the $\phi(f)$, which generate a maximal abelian algebra, so that $W(a)$ is the operation of multiplication by some function $K(a, x)$ on $L_{2}(G)$. It is easily verified from the group representation property of $U$ that $W$ satisfies the equation

$$
W(a b)=\left[U_{0}(b)^{-1} W(a) U_{0}(b)\right] W(b),
$$

from which the 'multiplier relation'

$$
K(a b, x)=K(a, b x) K(b, x)
$$

is valid for each pair $a$ and $b$ for almost all $x$. To complete the proof it suffices to show that $K(a, x)$ can be chosen so as to be measurable on $G \times G$, for $K\left(a, a^{-1} x\right)$ is then likewise measurable (being its transform under a measure-preserving homeomorphism), and the unitary operator $S$ consisting of multiplication by $K\left(x, x^{-1} a\right)$, where $a$ is chosen so that this is a measurable function, is readily seen to trans- 
form $U(a)$ into $U_{0}(a)$, for all $a$; the use of the Fubini theorem just made may be justified by the reduction to the case of a sigma-compact group as earlier.

Now $W(a)$ is a continuous function of $a$ in the strong operator topology, and is easily seen to have a 'direct integral' $K$ on the Hilbert space $L_{2}(G \times G)$, which may be defined by the equation

$$
(K f, g)=\int(W(a) f(a, \cdot), g(a, \cdot)) d a .
$$

This operator $K$ is easily seen to commute with multiplications of functions $f(a, x)$ by arbitrary (bounded measurable) functions of $a$ as well as by arbitrary functions of $x$, which generate the maximal abelian multiplication algebra of $G \times G$, so that $K$ is itself multiplication by some bounded measurable function $K(a, x)$, which may be identified with the multiplier previously introduced.

It is worth noting that no essential use is made in this measurability proof of the complex-valued character of $K(a, x)$; it is equally applicable to the case in which its values are in a commuting set of unitary operators on a Hilbert space. With this observation and the use of multiplicity theory, the more general imprimitivity theorem of Mackey [56] may be treated in the same way.

A direct consequence of the foregoing is the generalized von Neumann theorem, in the simple spectrum case.

CoROLlaRy 1. Let $G$ be a locally compact abelian group with topological character group (compact-open topology) $G^{*}$; let $U$ and $V$ be continuous unitary representations of $G$ and $G^{*}$ on the Hilbert space $H$, satisfying the relations

$$
U(a) V\left(b^{*}\right)=b^{*}(a) V\left(b^{*}\right) U(a) \quad\left(a \in G, b^{*} \in G^{*}\right),
$$

and such that the $U(a)$ generate a maximal abelian algebra of operators on $H$. Then $(U, V)$ is unitarily equivalent to the standard pair $\left(U_{0}, V_{0}\right)$ on the Hilbert space $L_{2}\left(G^{*}\right)$ and with

$$
U_{0}(a): f\left(x^{*}\right) \rightarrow x^{*}(a) f\left(x^{*}\right), \quad V_{0}\left(b^{*}\right): f\left(x^{*}\right) \rightarrow f\left(x^{*}+b^{*}\right) .
$$

For the proof, $G^{*}$ here plays the role of $G$ in the Scholium. $\phi$ is then the homomorphism which the generalized Stone theorem associates with the representation $U$, while the present representation $V$ plays the role of $U$ in the Scholium.

The generalized Plancherel theorem now asserts

Corollary 2. Let $G$ be a locally compact abelian group with topo- 
logical character group $G^{*}$. The mapping from $L_{1}(G) \cap L_{2}(G)$ which carries $f$ into the function $F$ on $G^{*}$ given by the equation

$$
F\left(x^{*}\right)=\int x^{*}(a) f(a) d a
$$

is isometric into a dense subset of $L_{2}\left(G^{*}\right)$.

Note first that the operators $U(a): f(x) \rightarrow f(x+a)$ as a totality have simple spectrum, for they generate the same ring of operators as their smoothed versions $\int U(a) f(a) d a$, with $f$, say, continuous and of compact support; these form, in fact, an associative algebra $\boldsymbol{A}$, which, together with the functional $E$ defined by the equation $E\left(\int U(a) f(a) d a\right)=f(e)$, forms an integration algebra; for any such, the multiplication algebra generated by the defining algebra $A$ has simple spectrum by the proof of the Theorem; finally, the map $f \rightarrow \int U(a) f(a) d a$ is an isometry from a dense subset of $L_{2}(G)$ onto a dense subset of $L_{2}(A, E)$, carrying the relevant operators on $L_{2}(G)$ into the algebra of multiplications by elements of $\boldsymbol{A}$ acting on $\boldsymbol{A}$. The preceding corollary is therefore applicable, and shows the existence of a unitary operator $F$ from $L_{2}(G)$ onto $L_{2}\left(G^{*}\right)$ which carries $U(a)$ into $U_{0}(a)$ and also the operation $V\left(a^{*}\right): f(x) \rightarrow a^{*}(x) f(x)$ into $V_{0}\left(a^{*}\right)$, for all $a$ and $a^{*}$. As noted by Nakamura and Umegaki [69], $F$ has the property that if $f$ and $g$ are any two elements of $L_{1}(G) \cap L_{2}(G)$, then $F(f * g)=\hat{f} \cdot F g$, where $\hat{f}$ is defined by the integral formula, from which it follows by substitution of an approximate identity for $f$ that $F g$ and $\hat{g}$ can differ only by a fixed multiplication operator, which is easily seen to be necessarily constant.

Thus the Scholium can be regarded as inclusive of the generalized Plancherel theorem in the case of an abelian group; it is, nevertheless, basically a commutative theorem even when the group is noncommutative, and, in this case, quite distinct from the generalized Plancherel theorem. This is visible in an elementary fashion in the case of a compact non-abelian group, the generalized Plancherel theorem then taking the form of the expansion of a general $L_{2}$-function on the group which is given by the Peter-Weyl theorem.

4. Integration in function space. In the preceding section algebraic integration theory appeared as an instrument for increasing the comprehensibility and for developing in a generally economical way a complex of results previously obtained without the use of the theory. In the theory of integration in function space, however, algebraic integration theory or some near equivalent appears as an essential part of the results as well as the method. 
A pure mathematician comes to the subject perhaps most readily from the standpoint of the problem of constructing an invariant function theory in a real Hilbert space which will as far as possible extend the classical theory in $n$-dimensional euclidean space. Most applied mathematicians and probabilists will more naturally proceed from the theory of stochastic processes, and, especially, of Brownian motion. Still another approach would be natural for the mathematical physicist-that of the theory of the free Bose-Einstein quantum field and of the representations of the canonical commutation relations. It would be well worth describing all of these approaches and delineating the nature of their ultimate substantial convergence. For brevity, however, a somewhat eclectic approach, conceptually one of the simplest, will be followed and the others suggested by the character of indicated applications in their respective directions

The question of the existence of a measure in Hilbert space which is invariant under translations has a fairly substantial history. While one would naturally like a direct generalization of euclidean measure in $E_{n}$, one would actually settle for much less; however, there is no possibility of such a settlement, since nothing of analytical viability which is translation-invariant appears to exist. A mathematician pondering this state of affairs might be struck by the fact that the isomorphism group of a Hilbert space is really the orthogonal rather than the translation group, and explore possible invariant integrals relative to this group. If, despite uniqueness problems, he persisted, he might try to pick out a particular invariant integral by a further requirement of an invariant nature. Now there is, indeed, one important and obvious property of both the euclidean and the isonormal elements of measure in a finite-dimensional space ('isonormal' will mean isotropic Gaussian centered at the origin, for brevity, unless otherwise specified). This is the independence, from an integrationtheoretic standpoint, of orthogonal subspaces. To be explicit, let a function $f$ on a Hilbert space be said to be based on a submanifold $M$ if it depends only on the component of the variable in $M$ (i.e., $f(x)$ $\left.=f\left(P_{M} x\right)\right) ; f$ is then determined by its restriction to $M$. Now if $M$ and $\boldsymbol{N}$ are orthogonal subspaces of a finite-dimensional Hilbert space $H$, and if $f=g h$, with $g$ based on $M$ and $h$ on $N$, then $\int f=\int g \cdot \int h$, evidently; and this simple property is evidently quite a useful one. Let us ask then: does there exist an orthogonally invariant integral on Hilbert space such that orthogonal manifolds are independent in the foregoing sense, and if so, to what extent is it unique?

In an infinite-dimensional space this requires the measure of the entire space to be one, since the function identically one on the space 
is based on every subspace. With this restriction there is a simple answer to the foregoing question: there exists no countably additive measure on $H$ of the type indicated; however, there is an invariant integral on the bounded tame functions of the type in question, and this is unique within multiplication of the associated weak probability distribution (i.e., of the associated linear random variables) by a constant. This is the isotropic, centered Gaussian integral on Hilbert space. Specifically, if $f$ is based on the finite-dimensional manifold $M$, then

$$
E(f)=c \int_{M} f e^{-Q} d x
$$

where $Q=c^{\prime}(x, x), x$ denoting a variable vector in $M$ and $c^{\prime}$ a positive constant, and where $c$ is a normalization constant.

At first glance this may seem quite a different object from that originally sought, which was to be translation invariant. It has, however, a property which the abstract harmonic analyst will recognize as being almost as useful for many applications-it is quasi-invariant. There is, to be sure, an elusive quality to this quasi-invariance; the familiar definition employed, e.g., in the last section, requiring invariance of null sets or relative absolute continuity of the integral and its translates is meaningless as it stands, in the case of integration algebras. But it may, nevertheless, be defined in a conceptually simple fashion, which extends the notion as it has been used on conventional measure spaces.

For generality, let $(A, E)$ denote an arbitrary integration algebra, and let $(A, F)$ denote another with the same algebra. Then $F$ may be said to be 'absolutely continuous' with respect to $E$ in case there exists an abstract non-negative measurable function, or, for short, 'measurable' $D$ relative to $(A, E)$ such that

$$
F(X)=E(X D), \quad X \in A .
$$

It may be asked: 'On which of the infinity of measure spaces affording concrete representations of $(A, E)$ is $D$ to be defined?' but the answer is simply 'It doesn't matter'; if the equation holds in any one space, then it holds in all, and the various 'derivatives' of concrete representatives for $F$ relative to concrete representatives for $E$ are carried into one another by the equivalences between the corresponding concrete spaces. Alternatively, these equivalences could be avoided by working in a completely abstract way, defining the measurables as the elements of the completion of $A$, or, more precisely, its quotient algebra modulo elements of vanishing square integral, in 
any of various topologies (all leading to complete linear spaces which are essentially the same as linear sets). While this is conceptually the simplest way, to carry out all the proofs without the use of representation theory would be largely to indulge in a lengthy paraphrase of conventional integration-theoretic procedures. A more concrete approach than this, while at the same time distinctly less circumlocutory than the first, is to represent $\boldsymbol{A}$ by the corresponding algebra of bounded operators on the $L_{2}$-space described earlier. The measurables may then be identified with the self-adjoint operators commuting with every element of $A$, and the product $X D$ with the closure of the usual product. The required extension of the domain of definition of $E$ may be obtained in a variety of ways; for example, by the preliminary extension of $E$ to all bounded linear operators in the ring generated by $\boldsymbol{A}$, followed by the extension to the non-negative unbounded self-adjoint operators whose spectral projections are in this ring (it is no essential alteration to limit the $X$ in the defining equation for $D$ to non-negative elements of $A$ ).

Adopting now any of these means of defining absolute continuity, an absolutely continuous transformation on the finite integration algebra $(\boldsymbol{A}, E)$ is naturally defined as an homomorphism $\chi$ of $\boldsymbol{A}$ into itself such that the transformed functional $F$ given by the equation $F(X)=E(\chi(X))$ is absolutely continuous relative to $E$. If there is given a group $G$ acting via automorphisms on $A$, a quasi-invariant integration algebra relative to this representation of $G$ may be defined as one such that the various functionals obtained by transformation of $E$ via the action of $G$ are mutually absolutely continuous. By the same argument as indicated in the preceding section, this is equivalent to the existence of a unitary representation $U$ of $G$ on $L_{2}(A, E)$ having the property that

$$
U(\chi) X U(\chi)^{-1}=\chi(X)
$$

for all the automorphisms corresponding to the elements of $G$ (which it is no essential loss of generality here to identify with the automorphism group itself). In practice, it may be a nontrivial problem to identify a convenient subalgebra of $L_{\infty}(A, E)$ which a given group of geometrical transformations leaves invariant through its induced action, and which determines the same integration algebra-this is especially the case with non-linear transformations, which will, in general, carry tame functions into nontame functions, and whose absolute continuity is thereby defined only if the transformed functions can be approximated sufficiently closely by tame or other amenable functions-but in the case of the group of translations on a Hilbert 
space, relative to the algebra of tame functions, say, there is no such problem, nor is there any even for the larger affine group. For any affine transformation $S: x \rightarrow T x+a$ on a linear space $L$, in fact, a given weak distribution $n$ on $L$-i.e., a linear map from $L^{*}$ to random variables (or rather an equivalence class of such) -is quasi-invariant (or the transformation is absolutely continuous relative to the distribution) according to this definition in case there exists a unitary transformation $U$ such that

$$
U n(f) U^{-1}=n_{S}(f),
$$

where $n_{S}$ is the weak distribution given by the equation $n_{S}(f)$ $=n\left(T^{*} f\right)+f(a)$.

In the case of the isonormal distribution on a Hilbert space, there is no great complication in writing down explicitly such unitary transformations for given translations. Now there is a distinguished unitary transformation corresponding to a given absolutely continuous transformation-a unique one which is the product of the induced automorphism of $\boldsymbol{A}$ with a non-negative factor-and this correspondence is, in fact, a group homomorphism. In the isonormal case there results in this way a unitary representation, say $U(\cdot)$, of the additive group of the Hilbert space, which is, moreover, continuous (in the strong operator topology). Denoting by $V(x)$ the unique unitary transformation which carries $n(f)$ into $e^{i f(x)} n(f)$ for all $f$, the pair $(U, V)$ then satisfies the 'Weyl relations'

$$
U(f) V(x)=e^{i f(x)} V(x) U(f),
$$

and so provides an analogue for the Hilbert space to that associated with a locally compact abelian group. They lead also to analogues to the important canonical $p$ 's and $q$ 's which operate on $L_{2}\left(E_{n}\right)$ by differentiation in a given direction and multiplication by a given linear functional; specifically, $P(x)$ and $Q(x)$ may be defined as the selfadjoint generators of the continuous one-parameter groups $[U(t x)$, $t$ real] and [ $V(t x), t$ real] (identifying the real Hilbert space with its dual). These satisfy the usual commutation relations:

$$
[P(x), P(y)]=0=[Q(x), Q(y)] ; \quad[P(x), Q(y)]=i(x, y) I,
$$

and there are many further analogies with the finite-dimensional euclidean situation, one of which will be described shortly. However, the use of the Gaussian integral has the effect that the restriction of the theory to the finite-dimensional case is merely readily transformable into, and not actually identical with, the classical theory. This shows up, for example, in the fact that $i P(x)$ is not simply the opera- 
tion of differentiation in the direction $x$, in the case, say, of its application to a smooth tame function, which is not self-adjoint as an operation on the Hilbert space in question, but differs from this differentiation through the addition of a simple multiplication operation, which is precisely that required to yield a self-adjoint operator without disturbing the commutation relations, and to satisfy the positivity requirement earlier indicated.

The theory of the Fourier transform is, on the other hand, basically parallel to the usual one. The utility of the Gaussian integral in the treatment of the Plancherel theory has long been known and is emphasized in Wiener's treatment [117]. Its fundamental role in the present situation carries with it substantial incidental simplifications that can best be understood in terms of unitary as opposed to mere orthogonal and translation invariance of the conventional approach (see below); among these are the elimination of an arbitrary scale factor in the expression of the Fourier transform, the convenience of working with the polynomial algebra which also makes possible the isolation of the purely algebraic aspects of the theory and facilitates the understanding of the 'Feynman integral,' and the elimination of the need for any nontrivial computations other than that of the integral $\int_{-\infty}^{+\infty} e^{-x^{2}} d x$. A quite closely related transform was introduced originally in Wiener space in the work of Cameron and Martin [9], and it seems appropriate as well as convenient to call the modified Fourier transform in Hilbert space the Wiener transform; when the space is finite-dimensional this transform is the similarity transform of the Fourier transform via multiplication by a Gaussian factor (which, however, becomes infinite with the dimension of the space).

The Wiener transform of a square-integrable functional on Hilbert space $H$ relative to the isonormal distribution may be defined by first defining the transform $W f$ of a polynomial $f$ by the equation

$$
W f(x)=E\left[f\left(2^{1 / 2} x+i y\right)\right],
$$

where the polynomial character of $f$ is made strong use of through the use of a complex argument for it; it is evident that $W f$ is again a polynomial, and demonstrable that it has the same $L_{2}$-norm, so that this transform may be uniquely extended to an isometric transformation on all of $L_{2}(H)$ which is, in fact, unitary, since the inverse of the indicated mapping on polynomials may be shown to exist, and to be represented, in fact, by the same expression with the substitution of a - for the + sign. The transformation $W$ has the virtually definitive property that

$$
W P(x) W^{-1}=Q(x), \quad W Q(x) W^{-1}=-P(x), \quad x \in H .
$$


Unlike the Fourier transform, $W$ is applicable to the function identically one and leaves it invariant; in quantum-field-theoretic language, incidentally, this means $W$ leaves the standard vacuum (state representative) invariant. The Fourier transform in function space is a natural tool for quantum field theory and in connection with certain stochastic processes. It is, however, a highly distinguished case of an important transform associated with an arbitrary unitary transformation on a complex Hilbert space, namely, that associated with multiplication by $i$.

Not only does the Gaussian integral provide an adequate substitute for many purposes to the euclidean volume element, it actually has a higher degree of invariance than the euclidean measure, but relative to a partially different group, this unitary group, which is asymptotically substantially higher in dimension than the combined orthogonal and translation groups. At first glance, the action of the unitary group on the functions over a real space is likely to appear baffling, but in a finite number of dimensions it is a special case of the action of the symplectic group indicated in the previous section. It is, however, an ordinary representation in the case of the unitary group rather than a projective one as in the case of the symplectic group.

To describe the action of the unitary group, let $H$ denote the real Hilbert space in question, and let $H^{\prime}=H+i H$ denote its complexification, so that every vector $z$ in $H^{\prime}$ has the form $x+i y$ for unique vectors $x$ and $y$ in $H$. To each unitary transformation $U$ on $H$ there is then a unitary transformation $\Gamma(U)$ on $L_{2}(H)$ which is determined within a scale factor by the property that it transforms the so-called 'canonical' $P(x)$ and $Q(y)$ in the natural fashion. This transformation rule may be expressed most simply through the introduction of the so-called 'creation' operators $C(z)$, which have the advantage of complex linearity; setting

$$
C(z)=\frac{1}{\sqrt{ } 2}(P(x)+i P(y)+Q(y)-i Q(x))
$$

the non-normal operator $C(z)$ is analytically troublesome but has convenient algebraic properties, including complex-linearity: $C(a z)$ $=a C(z)$ for any complex number $a$, and commutation relations which depend only on the complex Hilbert space:

$$
\left[C(z), C\left(z^{\prime}\right)\right]=0=\left[C(z)^{*}, C\left(z^{\prime}\right)^{*}\right],\left[C(z), C\left(z^{\prime}\right)^{*}\right]=\left(z, z^{\prime}\right)
$$

(for simplicity of exposition we are ignoring the presently irrelevant matters of closures of the unbounded operators involved here. The relations involving the $C(z)$ are obviously invariant under any iso- 
morphism of the complex Hilbert space, and it is natural that for any given such isomorphism-i.e., unitary transformation $U-$ there should exist a unitary transformation $\Gamma(U)$ on $L_{2}(H)$ which transforms $C(z)$ into $C(U z): \Gamma(U)^{-1} C(z) \Gamma(U)=C(U z)$. In fact, this property determines $\Gamma(U)$ within a constant factor; observing that $\Gamma(U)$ necessarily carries the unit functional on $H$ into a constant functional, it is naturally normalized so as to leave fixed the unit functional, and the map $\Gamma$ is then a unitary representation of the unitary group on $H . \Gamma(U)$ is familiar in two cases: (i) that in which $U$ is the extension to the complex space of an orthogonal transformation $O$ on the real space; in this case $\Gamma(U)$ is simply the induced action on $L_{2}(H)$ of this orthogonal (essentially measure-preserving) transformation, i.e., carries a given tame functional, say $f(x)$, into $f\left(O^{-1} x\right)$ and (ii) that in which $U$ is the direct sum of multiplication by $i$ on a certain subspace of $H^{\prime}$ with the identity on the orthocomplement of the subspace; in this case $\Gamma(U)$ is the Wiener (or transformed Fourier) transform relative to the variables forming coordinates for the vectors in the subspace.

The unitary group differs, of course, from the euclidean group in that it does not operate directly on the real space $H$ itself. Its action is, however, of fundamental importance both in quantum field theory and the general theory of Brownian motion. From a purely mathematical standpoint the surprising fact that such a relatively large group may be made to act on such a relatively small space, in a fashion extending the actions indicated in (i) and (ii), may be regarded as the group-theoretic clue to the remarkable utility which expansions into Hermite functions have found in a number of realanalytical situations in which symmetry considerations, although present, are rather hidden.

The most notable role of the Hermite functions in this regard has been in connection with the 'homogeneous chaos' of Wiener [116], whose utility in a variety of connections has been emphasized in later work by Wiener. It was developed further by Kakutani [48], who showed that the closure of the orthocomplement of the chaos of order $n-1$ in that of order $n$ was unitarily equivalent to the space of symmetric square-integrable functions over $E_{n}$. The unitary equivalence in question carried temporal displacement in the Wiener process, or rather, its induced action on square-integrable functionals, into a corresponding displacement $f\left(x_{1}, \cdots, x_{n}\right) \rightarrow f\left(x_{1}+t, \cdots, x_{n}+t\right)$ of the symmetric functions on $E_{n}$, enabling the completion of work of Wiener and Anzai on the spectrum of Brownian motion. This is a highly special case of a result giving the group-theoretic reduction of the representation $\Gamma$; only the abstract Hilbert space character of $L_{2}$ 
on the reals and the unitary character of the displacement $f(x)$ $\rightarrow f(x+t)$ are relevant. The proof of this more general result is actually simpler than that for the special case treated by Kakutani, which is clearly applicable only to the treatment of the restriction of $\Gamma$ to the cited one-parameter orthogonal group. Indeed, the unitary equivalence in question is far from unique; if formulated properly, however, it not only gives the explicit reduction of $\Gamma$ but serves to correlate the operators $C(z)$ with the so-called creation operators of Fock and Cook [12A], and because of the irreducibility of these operators is then unique, within a scalar factor. ${ }^{3}$

The group-theoretic structure of the representation $\Gamma$ and its restriction to the orthogonal subgroup are similar to their structures in the case of a finite-dimensional space as revealed in the classical work of Schur and Weyl on the reduction of induced tensor representations. The behavior of the representation of the full unitary group is particularly simple. The generator $N$ of the one-parameter group $\Gamma\left(e^{2 \pi i t} I\right)$ has integral proper values by virtue of the periodicity of the exponential function; it turns out that only non-negative proper values occur, each with multiplicity one. The corresponding eigenspaces are then invariant under $\Gamma$, and, as it turns out, irreducibly so; the subspace corresponding to the eigenvalue $n$ is unitarily equivalent (as far as the action of $\Gamma$ is concerned) to the action of the unitary group on the symmetric tensors of rank $n$ over the underlying complex Hilbert space $\boldsymbol{H}$. Parenthetically, let us note for those interested in relations with quantum field theory that the non-negative integral character of the generator $N_{P}$ of the groups $\Gamma\left(e^{2 \pi i t P}\right)$, where $P$ is an arbitrary projection on $H$, makes possible an interpretation of $N_{P}$ as 'the number of particles with wave function in the range of $P$, which interpretation is further substantiated by statistical features corollary to the group-theoretic properties of $\Gamma$.

In fact, useful as the representation $\Gamma$ and its reduction into irreducible constituents are in probability and flow theory, they are more fundamental in the quantum field theory. The abstract character of the underlying space $H$ is rather essential here because the underlying symmetry group does not act simply by point transformations on an $L_{2}$-space, but in a more complicated manner (cf., e.g., Segal [97]). The physical interpretation is mathematically illuminating in important respects. For example, the operator $N$ described in the preceding paragraph has no special significance from a probability viewpoint, appearing simply as a means of describing

8 In the later article [48A] this is taken advantage of to extend the reduction to the case of $\Gamma(O)$ for an arbitrary orthogonal $O$; the results here are, however, consequences of those cited, taken from [90] (applicable to $\Gamma(U)$ for arbitrary unitary $U$ ). 
certain subspaces; its interpretation as the 'number of particles' variable for a 'Bose-Einstein field' serves to rationalize the otherwise fortuitous non-negativity of the operator, and to suggest further interesting mathematical relationships. In physical terms, the representation $\Gamma$ is precisely that which transforms a 'free single-particle motion' $U$ into the corresponding motion of the quantum field consisting of an indefinite number of particles. Considerably more could be said on this subject, but we shall not interrupt the present basically purely mathematical exposition.

We have now arrived, in a preliminary way, at a partial, but ex post facto fairly natural, analogue to the euclidean integral for the case of an infinite-dimensional real Hilbert space, which compensates for its differences from the euclidean volume element with some distinctive uses of its own. Now one of the most important aspects of an integral from the standpoint of practical analysis is its behavior under transformations of the underlying space, other than those preserving the underlying geometry, which have already been discussed. The simplest and most basic case is that of a general nonsingular linear transformation, say $x \rightarrow T x$. In the finite-dimensional case this alters the integral only by a constant factor, the determinant of $T$; inasmuch as the determinant is undefined for general nonsingular transformations on a Hilbert space, there is some difficulty in extending this result to Hilbert space. There is, however, an important and natural class of operators having well-defined and well-behaved determinants, namely, those of the form $I+X$, where $X$ is an operator of absolutely convergent trace (cf. [99]). It is reassuring that, despite the replacement of the euclidean by the Gaussian volume element, there is a simple rule for the transformation of the integral corresponding to such a linear transformation on the space, which extends the usual one for a finite-dimensional space. More specifically, the transformation $T$ is absolutely continuous, so that there exists an abstract measurable $D(=D(T))$ such that

$$
\int_{H} f_{T}=\int_{H} f D
$$

for any tame function $f$, where $f_{T}$ indicates the transformed function $f(T x)$; in fact, a simple expression for $D(T)$ entirely analogous to the usual one for the transformation of the Gaussian integral in a finite-dimensional space may be given.

While this development is highly natural from a formal viewpoint, a rigorous development first appeared in quite another way, in the 
course of the exploration of the analytical properties of Wiener space by Cameron and Martin [8] some years before any effective exploration of the idea of integration in Hilbert space took place. For a general class of transformations defined by integral operators, it was shown that Wiener measure transformed in absolutely continuous fashion, and the Radon-Nikodým derivative was given in a closed form involving the determinant of the operator in question. The problem of the absolute continuity of linear transformations on Gaussian stochastic processes arises in a number of applications, and the question has continued to receive attention in other settings (cf. [78] and below). The Hilbert space result was established in [94], which also indicated the connection with the theory of Brownian motion. The proliferation of formalisms and the difficulty in applied work of dealing with isomorphisms has led to a good deal of misunderstanding of the relations between varieties of integrals in function spaces which is quite marked in connection with this relatively simple but important absolute continuity result for linear transformations, which may therefore be an appropriate point for a more explicit consideration of these matters.

The foreword to the work of Friedrichs and collaborators [25] states, in slightly paraphrased form: 'No attention is given to the work of I. E. Segal . . . ' While this could be (and was) construed as a concession to the needs of brevity and prompt publication, it was in a way surprising, inasmuch as most of the nontrivial results contained in these notes were already contained, apart from quite straightforward questions of specialization, in a different and more general formalism, in the indicated work. Later developments, however, made it rather clear that the nature of the relationship between the two 'theories' was quite obscure to the cited authors, and that it was in fact apparently believed that no significant rigorous mathematical connection existed (see especially [98] and below). Since our work had been available in advance of publication, and had been exposed verbally on several occasions (indeed, in part at the request of some of these authors), a need for a more thoroughgoing exposition of the relationship between different formalisms dealing with integration in function space was clearly indicated, and represents one of the purposes which this article hopes to accomplish. Although the essential mathematical inclusion of the formalism of the cited work as well as later work by some of the authors has now been apprehended (cf. the correction in [98]), the fundamental simplicity of the relations between the three best-known mathematical approaches to analysis in function spaces seems to remain somewhat obscure; in the recent 
fairly comprehensive and topical review article of McShane [65] (to whose useful bibliography on integration in function space we refer the reader for material supplementary to that cited here), it is again suggested that the developments described by Friedrichs and his associates are mathematically disjoint from the theory originated in [89] and developed initially chiefly in [90] and [94]-as well as from the theory of analysis in Wiener space.

There are basically two stages involved in the verification of the essential identity of integration theories, which it will suffice for present purposes to describe in the case of those corresponding to finite measure spaces: (i) the formulation of a convenient algebra of bounded abstract measurables which determine the full algebra (i.e., are dense in the $\mathrm{w}^{*}$-topology in $L_{\infty}$ ); (ii) the establishment of an algebraic isomorphism which is at the same time integral preserving.

Conditions on a subalgebra equivalent to its $\mathrm{w}^{*}$-density are that it be dense in $L_{2}$, or that it generate the full abstract measure ring for the space in question [89]; in practice, the first of these two conditions is at least as simple to establish as the others. Now in the case of Wiener space, formulated as the space $C_{0}[0,1]$ of all continuous functions on the interval $[0,1]$ which vanish at 0 , the algebra of all polynomials in the random variables $x(t)$ representing values of the Wiener process at arbitrary points $t$ in the interval forms such an algebra. These are, of course, unbounded functions, and to employ the foregoing general approach, it is necessary to approximate them by bounded functions. (In the case at hand, it would be possible to deal directly with the algebras of unbounded elements, but, in general, isomorphism of subalgebras of not necessarily bounded elements of $L_{2}$ would be insufficient to establish the essential identity of the associated integration theories.) This might suggest the use of the algebra of all polynomials in bounded functions of the $x(t)$, which is likewise dense in $L_{2}$, but for present purposes a slightly larger algebra is convenient. This is the algebra of all bounded Baire functions ${ }^{4}$ of finite ordered sets of the Wiener stochastic integrals $\int f(t) d x(t)$, where the functions $f$ are chosen arbitrarily in $L_{2}(0,1)$.

The mapping $f \rightarrow \int f(t) d x(t)$ from $L_{2}(0,1)$ into random variables is ready-made for establishing an isomorphism between the foregoing algebra and the algebra of bounded tame functions over $L_{2}(0,1)$ relative to the Gaussian distribution. This is the case because the weak distribution defined by the indicated mapping is precisely the Gaussian distribution itself. Thus, if $F$ is such a bounded tame func-

4 There would be no essential difference if continuous rather than Baire functions were employed. 
tion, so that $F(f)=\phi\left[\left(f, f_{1}\right),\left(f, f_{2}\right), \cdots,\left(f, f_{n}\right)\right]$ for some bounded Baire function $\phi$ on $E_{n}$ and some ordered set $f_{1}, f_{2}, \cdots, f_{n}$ of orthogonal functions in $L_{2}(0,1)$, then the mapping

$$
F \rightarrow \boldsymbol{\phi}\left[\int f_{1}(t) d x(t), \int f_{2}(t) d x(t), \cdots, \int f_{n}(t) d x(t)\right]
$$

is well-defined into the algebra defined at the end of the preceding paragraph, and the integral of $F$ relative to the isonormal distribution has an expression essentially identical to that for the integral of its transform over Wiener space; the material identity of the two integrals follows trivially. Since the mapping is an algebraic isomorphism, this means that Wiener space and a countably dimensional Hilbert space relative to the isonormal distribution are integration-theoretically equivalent. Any theorem on, or property of, one space which deals only with abstract measurables, i.e., does not depend on a specific choice of a concrete 'version' for a measurable, or function in the residue class defining the measurable, thereby implies immediately a corresponding result for the other space.

Which of the two spaces to employ, when either is possible, is a function of the tactical scientific situation. In general, the use of Wiener space is advantageous only when the problem is phrased in terms of Wiener space and troublesome nonlinearities are involved in the transition to Hilbert space. On the other hand, there are problems which may appear to be of the latter type, such as the determination of the spectrum of the flow of Brownian motion, but in which the additional structure involved in the use of a concrete Hilbert space and explicit transformations thereon turns out to be immaterial and, in fact, an encumbrance. It should be noted that many of the questions of the theory of stochastic processes concerning Brownian motion refer specifically to its formulation in terms of Wiener space, for example, such questions as the differentiability of sample functions, and for these it is to be expected that the simplest treatment will be in terms of this formulation.

The treatment of integration in Hilbert space due to Friedrichs has gone through a process of development whose final stage only clearly provides an integral of the type considered here. In [23] a section entitled 'Hermite functionals and integration over the Hilbert space' discusses the question of an integral in Hilbert space and defines, relative to any choice of orthonormal basis for the space, the notion of Hermite functional and an inner product between two such functionals; this inner product is the same as that obtained in the representation of a boson field given in the theoretical literature (cf., e.g., 
Dirac [14]) in terms of an assembly of harmonic oscillators. This is as far as the theory is carried here and the author remarks that it it is not entirely satisfactory and indicates that a forthcoming treatment, including, in particular, work of J. Milkman, will illuminate the parallel with the usual type of integration. In fact, inasmuch as the product of Hermite functionals is not again a Hermite functional, or linear combination thereof, there is no natural way to define the corresponding integrable, measurable, or $L_{p}$ functions for any $p \neq 2$. In the work of Milkman [65A] in elucidation of this approach it is shown that a certain associated measure on Hilbert space is countably additive on subsets which are direct sums with a fixed co-finitedimensional subspace; in the light of later developments it is visible that this result is tantamount to the countable additivity of the indefinite integral of $e^{-x^{2}}$ in a finite-dimensional euclidean space, which, of course, does not require a special proof; thus at this stage the relation of the theory to conventional theory of integration was still somewhat obscure.

The definitive statement appears in the later notes [25], together with the article [24] whose aim is to show the material orthogonal invariance of the theory despite the essential use of a particular basis of the Hilbert space for its formulation. In present terms, let $e_{1}, e_{2}, \ldots$ be a basis for the real Hilbert space $H$; the mapping of any vector into into its sequence of components carries $H$ into the denumerable direct product of lines, which is then endowed with the direct product of copies of standard one-dimensional Gaussian distributions; in probabilistic terms, the measure is obtained by assuming that the Hilbert space coordinates form a sequence of identically distributed independent random variables each of which has the standard Gaussian distribution. This is, of course, not a measure on the Hilbert space itself, but a functional on the Hilbert space which depends only on a finite number of coordinates determines in an obvious way a function on the associated direct product space, and can thereby be given an integral, which can be extended to more general functions by a process of approximation and completion.

It is evident that this is a theory definable by the algebra of all functions which depend only on a finite number of coordinates with respect to the given basis and are suitably restricted in growth, all bounded Baire such functions, for example, relative to the integral provided by the isonormal distribution in a finite number of dimensions. This shows that the integral is a sub-integral of the standard Gaussian integral treated earlier (i.e., from a conventional measuretheoretic standpoint, it can be regarded as based on the same con- 
crete measure space and measure function, but with a possibly smaller class of measurable sets), and the demonstration of its equivalence with this integral reduces basically to showing that the cited subalgebra, dependent on the basis $e_{1}, e_{2}, \cdots$, is $L_{2}$-dense in the space $L_{2}(H)$ previously defined. This fact, which, incidentally, serves to establish the orthogonal invariance of the Friedrichs theory, and whose demonstration is the main goal of [24], follows by entirely elementary approximations, e.g., as follows.

It is required to approximate in $L_{2}(H)$ an arbitrary bounded tame function, say $f$, on $H$ by a bounded Baire function of a finite number of the coordinates. Since, however, any function in $L_{2}$ over $E_{n}$ relative to the isonormal distribution may be approximated in $L_{2}$ by a finite linear combination of products of bounded functions of the single variables constituting coordinates relative to any orthonormal basis, it is sufficient to show that any such product, say $h$, may be approximated arbitrarily closely in $L_{p}$ for all sufficiently large $p$ by tame functions $h_{0}$ relative to the original given basis; the Hölder inequality then shows that the monomials in these functions may be approximated arbitrarily closely by the corresponding monomials in the $h_{0}$. Thus it must be shown that if $e^{\prime}$ is any vector in $H$, then $\left(x, e^{\prime}\right)$ may be approximated in $L_{p}$ for all large $p$ by a function of a finite number of the inner products $\left(x, e_{i}\right)$. Expanding $e^{\prime}$ in terms of the $e_{i}$, $e^{\prime}=\sum_{i} a_{i} e_{i}$, then $\sum_{i=1}^{n} a_{i} x_{i} \rightarrow\left(x, e^{\prime}\right)$ in $L_{p}$ for all finite $p$, since, for arbitrary $u$ and $v$ in $H$,

$$
\begin{aligned}
\|(x, u)-(x, v)\|_{p} & =\|(x, u-v)\|_{p}=(2 \pi k)^{-1} \int t^{p} \exp \left[-t^{2} / 2 k\right] d t, \\
& =c_{p} k^{p / 2} \rightarrow 0 \quad \text { as } k \rightarrow 0 ;
\end{aligned}
$$

here, the case $u=\sum_{i=1}^{n} a_{i} e_{i}$ and $v=e^{\prime}$ is in question.

Thus there are explicit natural isomorphisms between dense classes of functions in the theory presented in [25], in the Wiener space theory, and in that for the isonormal distribution in a Hilbert space, so that any result about integration over one space implies as corollaries corresponding results about integration in the other spaces. However, depending on the result, its statement and/or its proof may be significantly simpler in one formalism or the other. This may be illustrated by some examples of importance for applications.

The absolute continuity of translation in Hilbert space, relative to the isonormal distribution, does not imply that in $C[0,1]$ the map $f \rightarrow f+g$ is absolutely continuous, and, in fact, this is false. The map 
$f \rightarrow f+g$ in $L_{2}(0,1)$ carries the functional of $u,(u, f)$, into $(u, f)+(u, g)$, which is to say, in terms of the Wiener representation $x(t)$ of Brownian motion,

$$
\int f(t) d x(t) \rightarrow \int f(t) d x(t)+\int f(t) d G(t),
$$

where $G$ is the indefinite integral of $g, G(t)=\int_{0}^{t} g(s) d s$. In other terms, there exists an isomorphism of the set of all random variables on Wiener space which acts in the indicated fashion on the basic linear random variables indicated.

To rephrase the existence of an isomorphism $\phi$ of the algebra of random variables over a probability space as a result on the absolute continuity of a point transformation on the space, it is necessary to determine such a measurable point transformation $T$ which induces $\phi$, in the sense that $[\phi(f)(x)]=f(T x)$, for almost all points $x$ of the measure space and all measurable functions $f$. In a formal sense, $T x$ is the point whose characteristic function (i.e., of the set consisting of the point) is the image of the characteristic function of $x$; this is by no means strictly true, for general probability spaces, but it is true for all separable spaces admitting quite mild regularity properties (by virtue of results which, in a crude form, appeared in the work of F. Riesz 50 years ago; the results of many refinements appear in the rather general result due to Mackey [59]). It is, however, not at all necessary to appeal to this general theorem in the present or other linear cases; the relevant transformation $T$ is readily explicitly determined. It suffices if the foregoing relation between $\phi$ and $T$ holds for the generators of a dense subalgebra of $L_{2}$, for it then follows for the subalgebra algebraically, and then for sequential limits of the algebra, and ultimately all $f$. The indicated linear expressions in the $x(t), \int f(t) d x(t)$, form such a set of generators, and the required equality is indicated above for the transformation

$$
T: f \rightarrow f+G .
$$

Thus it is not a general displacement in $C_{0}[0,1]$ which corresponds to a general displacement in Hilbert space, but rather displacement through an element which is absolutely continuous and has a squareintegrable derivative.

The absolute continuity of vector displacements in Hilbert space relative to the isonormal distribution thus implies only the absolute continuity of such special displacements in $C_{0}[0,1]$. This, however, is all that is valid. Indeed, in the original work of Cameron and Martin [6] the stronger assumption is made that the displacement be by 
an absolutely continuous element whose derivative has bounded variation. The formula for the derivative which they obtained suggested that square-integrability of the derivative should suffice; however, this result was first obtained as a corollary to the Hilbert space result, in the fashion just indicated, following which it was obtained by direct analysis in Wiener space.

The form of the Radon-Nikodym derivative of the transformed distribution with respect to the original one may, however, be distinctly misleading on occasion, as the more complex example of a linear transformation shows. Let $T$ be a given linear transformation on the real Hilbert space $H$; then the general theory, as already indicated, shows that the transform of the isonormal distribution $n$ by (the induced action of) $T$, say $n_{T}$, is equivalent to $n$ in the sense of mutual absolute continuity if and only if $T$ is nonsingular and $T^{*} T$ differs from the identity operator $I$ by a Hilbert-Schmidt operator. When the difference is additionally of absolutely convergent trace, the Radon-Nikodym derivative has the form (when suitably interpreted in terms of limits of tame functions in the manner indicated earlier)

$$
(\operatorname{det} A)^{-1 / 2} \exp \left[-\left(\left(A^{-1}-I\right) x, x\right)\right], \quad A=T^{*} T .
$$

Since the determinant has an invariant unambiguous meaning only for operators differing from $I$ by an absolutely convergent trace operator, and since the quadratic functional $(B x, x)$ on a Hilbert space appears to define a random variable with respect to the isonormal distribution only when $B$ is of absolutely convergent trace (which, with $B=A^{-1}-I$, implies the same for $A-I$ ), it gives a specious indication for the necessity of this condition.

How the Hilbert-Schmidt condition comes to suffice may be seen more explicitly as follows. Suppose that $C=A^{-1}-I$ has the proper values $\lambda_{n}$ relative to the basis $\left\{e_{n}(n=1,2, \cdots)\right\}$, and that the $x_{n}$ are the coordinates relative to the latter; then, as random variables relative to the isonormal distribution, $x_{1}, x_{2}, \ldots$ form a sequence of independent identically distributed normal random variable of mean 0 and constant variance. If $C$ has absolutely convergent trace, then, by familiar probability theory, $\sum_{n} \lambda_{n} x_{n}^{2}$ is convergent almost everywhere to a well-defined random variable. On the other hand, assuming for simplicity that $C \geqq 0$, this is an if and only if proposition; the indicated series is convergent in any reasonable sense only if $\sum \lambda_{n}$ is convergent; otherwise $\sum_{n} \lambda_{n} x_{n}^{2}=\infty$ a.e. The actual absolute continuity in the Hilbert-Schmidt case is associated with the existence of a sequence of constants $c_{n} \rightarrow \infty$ such that the differences $\sum_{i=1}^{n} \lambda_{n} x_{n}^{2}$ 
$-c_{n}$ converge to a finite random variable, in terms of which the derivative may be expressed.s

In the case of the Wiener space theory, the corresponding result involves the determination of a point transformation $S$ in $C_{0}[0,1]$ which will induce the isomorphism which extends the mapping

$$
\int f(t) d x(t) \rightarrow \int T f(t) d x(t) .
$$

For simplicity of exposition, consider the case of a transformation $S$ of the form

$$
x(t) \rightarrow x(t)+\int_{0}^{1} K(t, s) x(s) d s,
$$

where $K$ is continuous on the unit square and, moreover, absolutely continuous as a function of $t$ for almost all fixed $s$, with a partial derivative $L(s, t)$ with respect to $t$ which is square-integrable. Then $L$ is a Hilbert-Schmidt kernel, so that the transformation

$$
T: f(t) \rightarrow f(t)+\int_{0}^{1} L(s, t) f(s) d s
$$

is absolutely continuous relative to the isonormal distribution. The identity

$$
\begin{aligned}
& \int_{0}^{1}\left[f(t)+\int_{0}^{1} L(s, t) f(s) d s\right] d x(t) \\
&=\int_{0}^{1} f(t) d t\left[x(t)+\int_{0}^{1} K(t, s) d x(s)\right]
\end{aligned}
$$

then implies by the same argument as in the case of vector translations the absolute continuity of the transformation $S$ on Wiener

- A physicist might say here that $\sum_{n} \lambda_{n} x_{n}^{2}$ exists and is well-defined apart from 'an infinite constant,' which is related to the infinite constant involved in the formation of the determinant of $A$ in such a way that there is cancellation, and the expression given for the derivative previously is still essentially correct. Mathematically, if the infinite product $\Pi_{n}\left(1+\lambda_{n}\right)$ is conditionally convergent, the same expression for the derivative applies and $c_{n}$ may be taken as zero. More generally, the Radon-Nikodym derivative is by the general theory the limit, both in $L_{1}$ and a.e., of the Radon-Nikodym derivatives of the corresponding restrictions to the subspaces spanned by $e_{1}, \cdots, e_{n}$, as $n \rightarrow \infty$, so that if $\operatorname{det} A_{n}^{-1}$ is convergent, where $A_{n}^{-1}$ is the finite-dimensional approximant to $A^{-1}$, then necessarily $\left(\left(A_{n}^{-1}-I\right) X_{n}, X_{n}\right)$ is correspondingly convergent, where $X_{n}$ is the projection of $x$ on the same subspace, to a measurable functional, and the expression for the derivative remains applicable. 
space. Again, the Hilbert space result, when transferred to Wiener space, proves more powerful than the original result due to Cameron and Martin [8], which is far from simple to formulate or to prove directly.

The question of absolute continuity of linear transformations relative to the isonormal distribution has also been considered somewhat later within the framework of the Friedrich notes [25], and represents probably the farthest point to which this framework has been applied. Unfortunately, in this work of Seidman [98], the result is not mathematically meaningful as stated, for reasons illustrative of some of the difficulties which tend to arise in the use of concrete countably additive measure-space models for weak distributions. This work involves a Hilbert space $H$, an orthonormal basis $\left\{e_{i}\right\}$, an embedding of $H$ in the space $l$ of all real sequences via the map $\sum_{i} x_{i} e_{i}$ $\rightarrow\left(x_{1}, x_{2}, \cdots\right)$, which space $l$ is endowed with the direct product measure corresponding to the element of measure $\left(2 \pi \sigma^{2}\right)^{-1} e^{-x^{2} / 2 \sigma^{2}} d x$ in each factor; the corresponding integral functional is denoted as $I$. The set $\boldsymbol{A}$ of operators is defined as those Hilbert-Schmidt operators for which the series $\sum_{i}\left(A e_{i}, e_{i}\right)$ is convergent, and $\Delta(A)$ denotes the determinant of $I+A$. The result as stated is as follows:

'Theorem. Let $A \in A$ and let $f$ be a function defined a.e. on l, measurable and integrable over $l$. Let

$E(x)=E(x ; A)=\exp \left[-(x, \Gamma x) / 2 \sigma^{2}\right], \quad \Gamma=\Gamma(A)=A+A^{*}+A^{*} A$,

and let

$$
g(x)=g(x ; A)=f(x+A x) \Delta(A) E(x ; A) .
$$

Then $E(x)$ is defined a.e. on $l, g(x)$ is defined a.e. on $l, g$ is measurable and integrable over $l$ and $I(g)=I(f)$.'

Now the indicated embedding of $H$ into $l$ maps it into a set of measure zero. If the values of $f$ on this null set are redefined as $0, f$ is not altered as a random variable, but $g$ becomes identically 0 on $H$. Thus the definition of $g$ is technically possible but there can be no relation between $g$ and the integral of $f$. The further statement that ' . . . is defined a.e. on $l$ ' becomes technically meaningful if altered to ' . . . is definable a.e. on $l$ ' but there is no indication in the statement as to how the extended definition is to be made, and, in view of the first comment, there is no possible means of extending the definition of $g$ so that the final equality, ' $I(g)=I(f)$ ' is, in general, correct.

From the vantage point of the general theory of the isonormal distribution in Hilbert space it is readily seen how to make a correct 
statement along similar lines. Because $A$ acts only on $H$ and not on $l$, and because $\boldsymbol{H}$ is a set of measure zero in $l$, it is necessary to take $f$ as a functional on $H$ rather than $l$ (if lengthy and doubtfully useful complications and circumlocutions are to be avoided). At the same time, $f$ must have unique integration-theoretic properties, so that it must either be a tame function or one approximable integrationtheoretically by such, and there is no real advantage in the employment of the latter, for an equality of the form ' $I(g)=I(f)$ ' for all tame $f$ will imply a corresponding one for all integrable $f$. Assuming, then, that $f$ is a tame function on $H$, and $E(x)$ and $g(x)$ are defined in the indicated fashion on $H$, it is meaningful to assert that these are measurable with respect to the isonormal distribution, in a sense which must further be specified through approximation by tame functions, and that $g$ and $f$ have the same integral over $H$. In this statement the role of $l$ has, however, been virtually eliminated; its introduction is merely a corollary to the result just indicated together with the earlier-indicated integration-theoretic equivalence between the isonormal distribution on $H$ and the cited distribution on $l$; it is not even possible to state the result in question without, in essence, including the statement for the isonormal distribution. ${ }^{6}$

Thus the absolute continuity of the measures in question under suitable linear transformations is readily stated for the case of Wiener space as well as for the isonormal distribution but can be asserted in the space $l$ only through a highly tautological procedure essentially requiring the transference of the result from some other model for the measure space. This should not be surprising since, where rather general linear transformations occur, the use of a fixed basis tends to lead to complications.

The abstract Hilbert-space approach has a clear advantage in the treatment of the absolute continuity question, as well as, in general, as regards conceptual simplicity and broad applicability, but special care and ingenuity are required for its effective use because of the countable additivity problem. On the other hand, the utilization of the sequence space $l$ is, in essence, the point of departure for the treat-

- To what extent the argument given in [98, I] supplies a proof of the present reformulation is not entirely clear; it appears to include some elements of such a proof. The later article [98, II], which appeared since the completion of the present exposition, proceeds generally along lines indicated above (as well as in our correspondence with the author); it seems correct, apart from its indication of greater generality in the expression for the derivative; the 'abstract' expression applies to the general case when suitably interpreted, as indicated in footnote 5; a special interpretation is required also in the 'concrete' case treated in [98]. 
ment in [72] of Wiener space, and there are few if any problems of a more sophisticated sort where it appears to have an advantage over either of the other two models. Wiener space itself is the natural model for employment in situations structured additionally by a special role for the Laplacian; similar 'sample' spaces are natural where a distinguished second-order linear elliptic differential operator occurs. This is the case, in particular, in the theory of the functional integral representations for the elementary solutions of the Schrödinger and heat equations, and variants thereof. The application of probability methods to the theory of differential equations has frequently been illuminating, and is interesting in itself in connecting two superficially independent disciplines. Functional integral solutions of integral equations were given first by Cameron and Martin [7], employing Wiener space; later, Feynman gave a quite heuristic approach to the Schrödinger equation involving complex probabilities; a formally similar development employing real probabilities associated with Wiener space was made later by Kac, and put on a quite rigorous basis by Ray (cf. [45]). More recently, Cameron, Ito, Babbitt, Feldman, Nelson and others have considered in a rigorous way the original situation discussed by Feynman. The two lastnamed authors have obtained thereby results of interest and power from a real-variable viewpoint concerning operators of the form $\Delta-V$, where $V$ is a given potential which may be so singular that the domain in $L_{2}$ of $\Delta$ and multiplication by $V$ have only 0 in common; remarkably enough, many such formal operators have natural interpretations as self-adjoint operators in Hilbert space. On the other hand, in the course of this work it has become apparent that what is basically involved is analytic continuation on a complex parameter on which the differential operator in question may be made to depend, rather than the utilization of a new integral.

The so-called 'Feynman integral' is, in fact, despite the virtual mystique which has grown up around it, from the abstract Hilbertspace standpoint, a very simple variant of the integral associated with the isonormal distribution. The isonormal distribution with variance parameter $\lambda$ is defined by a certain positive linear functional $E_{\lambda}$ on the algebra $P$ of all polynomials over the Hilbert space $H$, where, specifically,

$$
E_{\lambda}(f)=(2 \pi \lambda)^{-(n / 2)} \int_{M} f(x) e^{-x^{2} / 2 \lambda} d x,
$$

where $M$ is any finite-dimensional subspace on which $f$ is based and $n$ is the dimension of $M$. It is clear that $E_{\lambda}(f)$ is a polynomial in $\lambda$ for 
any fixed $f$, which is trivially applicable to all complex values of $\lambda$. The 'Feynman integral' with variance parameter $\lambda_{0}$ is simply the isonormal integral with variance parameter $i \lambda_{0}$, computed in the indicated fashion for any polynomial functional, and obtainable for other suitable functionals by approximation with polynomial functionals. In particular, if $f$ is a bounded tame function, the integral defining $E_{\lambda}$ is convergent if $\lambda$ has positive real part, and represents a holomorphic function of $\lambda$ in the right half-plane whose boundary values on the imaginary axis, if continuous, give more explicitly the Feynman integrals of $f$ for variable variance parameters as thus defined.

While the relation of the Feynman integral to the Wiener integral is basically equally close, Wiener space loses one of its chief advantages when employed as a model for the (indefinite) functionals $E_{\lambda}$ in that, as first noted by Cameron, the corresponding complex measure is not countably additive in Wiener space. On the other hand, the Brownian motion intervenes directly in the functional integral representing the elementary solution for the differential operator $\Delta-V$, which is given by the expectation values of expressions of the form $e^{-S V(x(t)) d t}$, where $x(t)$ indicates the Wiener Brownian motion. The formal analysis is thus naturally carried out in Wiener space, although the analyticity of this expectation value as a function of $\lambda$ for suitable $V$ is more easily understood in the abstract Hilbert-space setting. The integral $\int V(x(t)) d t$ is approximable by Riemann sums representing tame functions, which remain tame after substitution in the exponential, and so have expectations which are holomorphic functions of $\lambda$ in the right half-plane. But, as noted, the Feynman integral has been more of a stimulus than a tool in the cited work of Feldman and Nelson, who use Wiener space in establishing a one-parameter semigroup, and then continue analytically on the parameters on this semi-group in a basically operator-theoretic spirit.

For applications to nonlinear quantum fields, as well as nonlinear systems of a stochastic character, the transformation properties of functional integrals under nonlinear transformations are important. The first work on this question was that of Cameron and Martin [11], whose work may be qualitatively summarized as follows: if the nonlinear transformation on Wiener space is infinitesimally (i.e., if its Fréchet differential is) of an absolutely continuous character, and if there is adequate smoothness and uniformity, then the nonlinear transformation itself is absolutely continuous. The counterpart to this for the isonormal distribution remains true, as shown by Gross [39], but requires delicacy in its formulation since a nonlinear transform of 
a weak distribution is not automatically defined. In the case of a strict, countably additive distribution, the measure of the inverse image provides, as is well known, an appropriate and viable definition of the transformed distribution. But the image of a weak distribution under even the smoothest type of nonlinear map need not be definable in any generally effective way, as is shown by the case (to which the general case is reducible) of a map into a one-dimensional space, i.e., of a numerical function. For example, the function $e^{i(x, x)}$ on a Hilbert space is infinitely differentiable, even analytic in most reasonable senses, but meaningless as a random variable relative to the isonormal distribution because, as noted earlier, $(x, x)$ corresponds to the random variable which is identically $+\infty$.

Recourse to approximation by tame functions is again necessary; in general, there is no unique answer to the question of the randomvariable interpretation of a given smooth function on a linear space, relative to a given weak distribution theorem, except for tame functions. There exist a certain number of ad hoc procedures for treating special classes of functions, but these special results are in all known cases identical with those obtained from approximation with tame functions. For example, if $A$ is a self-adjoint operator of absolutely convergent trace on the Hilbert space $H$, the functional $(A x, x)$ corresponds to the random variable $\sum_{n} \lambda_{n} \tilde{x}_{n}^{2}$, where the $\lambda_{n}$ are the proper values of $A$ and the $x_{n}$ are the coordinates in $H$ for the corresponding proper vectors; and the superposition of the tilde $\sim$ indicates the structuring of $x_{n}$ as a random variable relative to the isonormal distribution. This represents it as a limit, in both the classical and integration-theoretic senses, of the tame functionals representing the partial sums of the indicated series. The form of this simplest measurable nonlinear functional and also of the derivative in the case of linear transformations suggest a special role for absolutely convergent trace operators, which has been well substantiated in the effectiveness of the topology in Hilbert space definable in terms of these operators introduced by Gross. Functions with suitable uniform continuity properties relative to this topology are, indeed, limits of sequences of tame functions in both classical and integration-theoretic senses, and correspond thereby to unique random variables, relative to any 'bounded' weak distribution, i.e., distribution $d$ on Hilbert space $H$ such that $\|d(x)\|_{L_{2}} \leqq$ const $\|x\|$. The generalization of these results in [40] clarifies and provides a practical solution in a wide class of cases to the problem of interpreting a given smooth functional as a random variable relative to a weak distribution. A clear general exposition is given in [42]. 
Following shortly after the beginnings of the work described on the theory of weak distributions in Hilbert space, intensive developments in related directions took place in the Soviet Union. The work of Gelfand and his collaborators is largely concerned with measures in relation to nuclear spaces, and is crowned by the characterization of such spaces as those for which any continuous weak distribution (=generalized random process in the sense of Gelfand) determines a countably additive measure (on the dual). A comprehensive account of this work is given in [33]. The work of Sazonov [81], Prohorov [76] and other probabilists are in the direction of applications to the theory of stochastic processes, rather than to relativistic physics. There is again an emphasis on the formulation of suitable spaces for the representation of a given weak distribution as a countably additive measure, and on the analysis of countably additive probability measures in function space. Group-invariance features in function space tend to become lost when countable additivity is insisted on, and the principal application to quantum field theory made is to the treatment of the representation problem for the canonical commutation relations made in [33]; this is generally similar to the treatment of the same subject given in [94].

The cited Gross topology in Hilbert space was employed by Sazonov [81] shortly after the completion of Gross's dissertation (at the University of Chicago) to characterize the Fourier-Stieltjes transform of a countably additive probability measure on Hilbert space by the usual positive-definite conditions together with continuity in the Gross topology. The most recent work in this direction is the extension of the Levy continuity theorem by Gross [41] to Hilbert space, from which the Sazonov result is readily deducible. Results on analysis in Hilbert space are also deducible from the nuclear space developments in [33] and, conversely, many of the results on analysis in general locally convex linear topological spaces, within which context the Soviet school tends to work, may be readily reformulated in terms of weak distributions in Hilbert space. In fact, a nuclear space is defined by a sequence of mappings by operators of absolutely convergent trace, and so implicitly involves Hilbert space and relates to the Gross topology. The nuclear space viewpoint is, of course, related to the use of distributions in the sense of Schwartz, and is involved also in some recent American work.

There are important applications of the foregoing developments to quantum field theory, although many significant questions involving functional integration remain unresolved; one of these is indicated in the introduction. 
5. Integration on operator rings. The algebra-cum-positive linear functional approach to in tegration theory is naturally extended, from a mathematical viewpoint, to the consideration of algebras which are not necessarily commutative. In this case algebraic experience suggests the enrichment of the structure by the postulation of an adjunction operation $a \rightarrow a^{*}$ for the algebra with the property that $E\left(a^{*} a\right) \geqq 0$ for the integral (functional) $E$. Actually, specific systems of this type arise frequently in functional analysis. Both from the concrete and the theoretical side, the additional assumption that $E$ be 'central,' i.e., that $E(a b)=E(b a)$ for arbitrary $a$ and $b$, plays an important role. It is a prominent feature of many important systems, and appears also as the crucial additional assumption needed for the extension of the abstract Lebesgue integration theory to what appears essentially to be its natural algebraic limits.

To describe the noncommutative theory, let $\boldsymbol{A}$ be a complex associative algebra with an adjunction operation * (i.e., involutory antiautomorphism), and let $E$ be a complex linear functional (called the integral in the Lebesgue context, and the trace in the operatortheoretic one) with the properties:

$$
E\left(a^{*} a\right) \geqq 0, \quad E\left(a^{*} a\right)=E\left(a a^{*}\right), \quad E\left(a^{*} b a\right) \leqq \text { const }_{b} E\left(a^{*} a\right)
$$

for arbitrary $a$ and $b$ in $\boldsymbol{A}$; then $(\boldsymbol{A}, E)$ may be called a (noncommutative) integration algebra. To remove the possibly singular part of the algebra in which all products vanish, which is devoid of interest, it is desirable to add the assumption that $\boldsymbol{A}^{2}$ is dense in $\boldsymbol{A}$ in the normtopology with the norm $\|a\|=\left(E\left(a^{*} a\right)\right)^{1 / 2}$, and it will be assumed in the following that the algebra is nonsingular in this sense.

Any self-conjugate subalgebra of the bounded integrable functions on a conventional measure space, with $E$ as the integral and the ${ }^{*}$ as complex conjugation, provide an example of an obvious sort. Conversely, the theory described earlier shows that any commutative integration algebra has this form, and that, moreover, the measure space in question is essentially unique, if the algebra is required to be dense in $L_{1}$. The simplest noncommutative example is a simple complex matrix algebra with $E$ defined as the trace and ${ }^{*}$ as hermitian conjugation. This is essentially the simplest instance of the 'factor' (= central simple operator ring $^{7}$ )-dimension function system treated by Murray and von Neumann; the dimension function, although strictly defined only for projections, may be extended by linearity to

7 'Operator ring' is used in this section to mean a 'ring' in the sense of Murray and von Neumann, i.e., a weakly closed self-adjoint algebra of linear operators, on a complex Hilbert space, which includes the identity. 
a linear set of operators which, in the matrix case, includes all operators. In brief, any factor has defined on its projections a completely additive function with values in $[0, \infty]$ which is unitarily invariant and unique within proportionality if some slight further condition to exclude trivial cases is imposed; this function is called the dimension function for the ring. Its construction is similar to that of Haar measure, with the notion of congruence replaced by that of 'equivalence' of projections relative to a ring-where this is not, in general, the same as unitary equivalence, which is not additive, but may be described as the minimal coarsening of unitary equivalence which is such, and is, indeed, completely additive and has the corresponding property that projections have the same dimension if and only if they are equivalent. For example, in the case of the ring of all bounded linear operators on Hilbert space, the dimension of a projection equals the conventional dimension of its range (i.e., cardinal number of an orthonormal basis for it-where infinite cardinals may be replaced by $\infty$ to obtain a function of the type indicated below). Those operators whose range is of finite dimension form an algebra $\boldsymbol{A}$ to which the dimension function may be uniquely extended by linearity and positivity to yield a functional $E$ satisfying the indicated conditions. Another example, which will be used later, is that in which $\boldsymbol{A}$ is the convolution algebra of all continuous functions of compact support on a unimodular locally compact group, with $E(f)$ defined as the value of $f$ at the group unit.

To continue with the general theory, the space $L_{2}(A, E)$ is readily defined as the completion of $A$ relative to the norm: $\|a\|_{2}=\left(E\left(a^{*} a\right)\right)^{(1 / 2)}$; the space $L_{1}(A, E)$ may be similarly defined, following the development of properties of the norm $\|a\|_{1}=\sup _{\|b\|_{\infty} \leq 1}|E(a b)|$, where $\|b\|_{\infty}$ denotes the usual bound for the operator $a$; analogous $L_{p}$ spaces may also be defined. However, this is not very satisfactory; there is no explicit interpretation analogous to that derived from the use of the unbounded measurable functions in conventional integration theory to represent points of the completion-which means, for example, that it is, prime facie, undetermined when two elements of different $L_{p}$ spaces are the same-and, in general, this approach provides too shallow a base for the construction of an effective analogue to the abstract Lebesgue theory. It is perhaps most logical to begin with the treatment of the concept of 'measurable' element of the extended integration algebra defined by $(A, E)$, designed so as to play the same role as the measurable functions in the conventional case and to reduce essentially to them in that case. Such measurable elements 
could presumably be introduced as elements of the completion of $\boldsymbol{A}$ relative to such a topology as convergence almost everywhere; while there are no actual points in a noncommutative measure space, this mode of convergence can be extended to the noncommutative case essentially by turning the criterion provided by Egoroff's theorem into a definition. Such a development has, however, never been carried out in detail, because it turns out to be possible to take advantage of operator theory in Hilbert space to introduce measurable elements as unbounded operators, in a way suggested by the earlier treatment of the commutative case; extreme methodological purity is thus sacrified for substantial technical advantages.

The well-known elementary algebraic difficulties concerning unbounded operators in Hilbert space, which might seem overriding, or at least to limit strongly the effectiveness of such a theory, turn out to disappear, essentially, within the domain of measurable operators. This circumstance can be regarded as a natural extension of similar circumstances in the special case of $\mathrm{II}_{1}$ factors treated in [68] and in commutative spectral theory. It should, perhaps, be mentioned, however, that the facility with which unbounded measurable operators may be treated does not at all resolve the old questionwhich is probably not effectively resoluble at all, according to present indications-of a unique calculus of variable coefficient differential operators in Hilbert space; in general, such operators are associated with rings which are isomorphic to all bounded operators, for which the only measurable operators are the bounded ones; on occasion, however, nontrivial Hilbert-space properties of differential operators are derivable from their measurability relative to other types of rings, as in [95] (for the case of integral operators, see below).

For any ring of operators $R$ there is a notion of equivalence of projections defined in the same way as in the case of a factor, as well as the notion of a 'finite' projection, defined as one equivalent to no projection which it bounds other than itself. The concept of a 'strongly dense' domain in the Hilbert space $H$, relative to the ring $R$, is definable in these terms as one containing an increasing sequence of closed linear subspaces $K_{n}$ such that the corresponding projections $P_{n}$ belong to $R$ and have finite orthocomplements $I-P_{n} \rightarrow 0$. For example, in the case of the multiplication algebra of a conventional measure space, if $\left\{S_{n}\right\}$ is any sequence of measurable subsets exhausting the space, the domain in $L_{2}$ consisting of all elements supported by some $S_{n}$ is strongly dense. In contrast to the situation for dense subsets, the intersection of two strongly dense subsets cannot 
consist only of the zero vector, but is, in fact, again strongly dense; indeed, the same is true of a countable intersection of strongly dense sets.

A measurable operator relative to a given ring $R$ may now be defined as a closed operator $T$ with a strongly dense domain, which is 'affiliated' with $\boldsymbol{R}$ in the sense, e.g., that it commutes with every unitary operator which commutes with every element of $R$. (Equivalently, $T$ is affiliated with $R$ if the partially isometric constituent and the spectral projections of the self-adjoint constituent in its polar decomposition are in $R$.) A sequence $\left\{T_{n}\right\}$ of measurable operators relative to $R$ may be said to converge 'almost everywhere' to a closed operator $T$ in case for every $\epsilon>0$ there exists a sequence of projections $P_{n}(\epsilon)$ in $R$ such that: (a) the operator $\left(T_{n}-T\right) P_{n}(\epsilon)$ is bounded by $\epsilon$; (b) $I-P_{n}(\epsilon)$ is finite and $\downarrow 0$ as $n \uparrow \infty$. This extends the usual notion on a conventional measure space - for which, as already indicated, the measurable operators are essentially the measurable functions (i.e., the measurable operators relative to the multiplication ring are the multiplications by such). In terms of this definition, the measurable operators may alternatively be defined as the limits under almost everywhere convergence of the simple operators, in an appropriate sense, in further close analogy with the conventional case. The central facts about measurable operators may be summarized in the statement that they form a topological ring with an involution, relative to the 'star' topology associated with convergence a.e.; this statement is for rings satisfying a certain weak countability condition, to which, in practice, more general rings may always be reduced.

The measurable operators are then entirely independent of the measure, being dependent only on the ring $R$, just as in conventional measure theory measurability is independent of the measure function and depends only on the sigma-ring on which it is defined. To return to integration proper, let us suppose given, in analogy with conventional measure theory, a completely additive, unitarily invariant non-negative functional on the projections in $R$-for short, a 'gage.' (In conventional theory, the measure, although only countably additive on a sigma-ring, induces a completely additive measure on the factor-Boolean-algebra of all measurable sets modulo the ideal of null sets; it is this factor algebra which corresponds to the algebra of projections in $R$, rather than the algebra of sets itself.) The integral may then be defined in an obvious way for finite linear combinations of projections of finite gage (although it is technically very difficult to establish the uniqueness of the extension by linearity-i.e., that if a linear combination $\sum_{i} a_{i} P_{i}$ of projections in $R$ vanishes, then so also does the putative integral $\sum_{i} a_{i} m\left(P_{i}\right)$, where $m$ is the gage- 
function). Because the set of all such linear combinations is not closed under multiplication when $R$ is noncommutative, it is more convenient to proceed somewhat differently, and define the integral on the ring $E$ of the elementary operators in $R$ consisting of those whose range is contained in that of a projection of finite gage. This may be done in various ways, but most commonly by defining the integral of the self-adjoint elementary operator $T$ with spectral resolution $\int \lambda d E_{\lambda}$ essentially as $\int \lambda d m\left(E_{\lambda}\right)$; the uniqueness difficulty cited earlier then appears as a difficulty in establishing additivity. The argument given by Murray and von Neumann in [67] to this end for $\mathrm{II}_{1}$ factors is one which extends readily, but it is one of the most involved in their series of papers; it is plausible that there should exist a basically simpler argument, but none is known. ${ }^{8}$ Actually, in practice, the gages which occur are associated with explicitly given functionals whose additivity is clear, except in the one instance of the dimension function. From the standpoint of applications the difficulty may therefore be regarded as primarily one in the theory of the dimension function rather than in integration theory proper.

At this stage there has been obtained, in one way or another, a central positive linear functional $E$ on a dense ideal, that of the elementary operators, $E$, in the ring $R$ (the density comes from the further assumption, which is no essential loss of generality as far as integration theory is concerned, that only the zero projection has vanishing gage). An integrable operator $T$ may now be defined as one which is integrable, and which is the limit almost everywhere of a sequence of elementary operators, $\left\{T_{n}\right\}$, which is a Cauchy sequence relative to the $L_{1}$ norm. The latter may be defined, on elementary operators, by the equation

$$
\|S\|_{1}=\sup _{\|X\| \leq 1}|E(S X)| .
$$

${ }^{8}$ The argument depends on unitary invariance, but it may plausibly be conjectured that any positive functional on a factor which is completely additive on commuting elements is universally additive, if the linear dimension of the factor exceeds 4 (or, correspondingly, on rings, if they include no so-called type $I_{2}$ component, which is exceptional). On the other hand, the argument employed by Gleason [35] to establish this, in the simplest nontrivial case of the ring of all bounded operators, is again notably intricate and apparently incapable of extension. These circumstances might suggest the existence of some fundamental difficulty if not the unsoundness of the conjecture. However, major unanticipated simplifications in the von NeumannMurray factor theory were made by Dye [19] regarding their trace formula and Griffin [38] concerning the spatial classification of type III rings, so that their treatment cannot be regarded as definitive. The discussion of the additivity question by Kadison [46] provides a simplified and improved exposition of the Murray-von Neumann argument. 
This definition is analogous to a familiar one in conventional measure theory. The proof of the uniqueness of the integral requires some delicacy but is basically parallel to that applicable in the conventional case. On the other hand, the proof of the positivity of the integral is relatively arduous, in distinction to its triviality in the conventional case. It might, therefore, be attempted to approach the integral differently, defining the integral of a positive measurable function, for example, as the supremum of the integrals of the positive elementary operators which it bounds. The problem of establishing additivity is, however, then of at least comparable difficulty to the proof of positivity with the first indicated approach.

The main formal properties of the integral then follow. There are effective extensions to the convergence theorems, the construction of the $L_{p}$ spaces, etc., from the conventional to the present case. While novel and substantial difficulties arise in establishing the noncommutative extensions of the classical theorems of Riesz-Fischer, RadonNikodym, Riesz, etc., they are all valid. More specifically, for example, (a) the $L_{p}$ spaces are complete; (b) the indefinite integral of an integrable operator may be characterized by a simple continuity property which is equivalent to that of countable additivity (and absolute continuity) in the conventional case; (c) the dual of the space $L_{1}(R, m)$ of all integrable operators is canonically isomorphic with $L_{\infty}(R, m)$, which is to say with $R$ itself. On the other hand, it is far from true that naturally conjectured extensions to results in the conventional theory have virtually all been established; even in the simplest nontrivial case, that of the ring of all bounded operators, some remain unresolved.

To consider in a more concrete way the situation in this ring $B$, the general hermitian element $T$ of $L_{p}(B)$ for finite $p$ is a compact operator, so that there exists a basis $\left\{e_{n}\right\}$ such that $T e_{n}=\lambda_{n} e_{n}$, where the $\lambda_{n}$ are the proper values of $T$, and such an operator is in $L_{p}(B)$ if and only if $\sum_{n}\left|\lambda_{n}\right|^{p}<\infty$, this sum being identical with $\|T\|_{p}^{p}$. Now if $T$ is integrable, its indefinite integral is the functional $F$ on $B$ defined by the equation

$$
F(X)=\operatorname{tr}(X T),
$$

where 'tr' is used to signify the trace, which is the integral in this case. The Radon-Nikodym theorem as extended then states that if, conversely, $F$ is a linear functional on $B$ which is strongly continuous relative to the unit sphere - the necessity of which property is easily seen-then it has the foregoing form. The Riesz theorem correspondingly states that every continuous linear functional on $L_{1}(B)$ has the 
form $X \rightarrow \operatorname{tr}(X S)$ for some element $S$ of $B$, whose bound equals that of the functional. This and some related results for the ring $B$ were obtained by Dixmier [15] shortly before the development of the general theory [88]. However, as an example of a simple natural question which remains unresolved even in this particularly simple case, consider the notion of the indefinite integral of a non-negative selfadjoint operator $V$, which may be defined as the completely additive functional $v$ on projections given by the equation $v(P)=\operatorname{tr}(V P)$ (or $+\infty$ if $V P$ does not have absolutely convergent trace in the usual sense). (This differs slightly but quite inessentially from the definition which is more convenient for the treatment of integrable operators.) Is it true, conversely, that every completely additive functional on the projections in $B$ is the indefinite integral of a nonnegative self-adjoint operator? Only when the values are all finite is the answer known to be affirmative, although in conventional integration theory this supposition is not needed.

A much less simple case, although the simplest of all factors other than $B$, is the so-called 'approximately finite' $\mathrm{II}_{1}$ factor of Murray and von Neumann. It may be defined as a rather natural type of limit of matrix algebras, more specifically as follows. First, any directed system of integration algebras has a direct limit which is again an integration algebra, by a basically straightforward extension of the general topological notion of direct limit to the situation at hand. Now consider the following directed system of matrix algebras: $\boldsymbol{A}_{n}$ is the ring of all complex matrices of order $2^{n}$, and is mapped into $A_{n+1}$ by the isomorphism

$$
A \rightarrow\left(\begin{array}{ll}
A & 0 \\
0 & A
\end{array}\right) .
$$

Let $A_{n}$ be formulated as an integration algebra relative to the trace, which is, indeed, the only gage-functional on $A_{n}$, since it is simple. If the trace is normalized in the usual way, so that $\operatorname{tr}(I)=2^{n}$, where $I$ is the identity operator in $A_{n}$, then, as would be expected, the direct limit is naturally identifiable with the integration algebra for $B$. If, however, the trace is normalized so that $\operatorname{tr}(I)=1$, then, in the limit, $\operatorname{tr}(I)=1$, so that the limiting integration algebra cannot be $B$. It is not difficult to see that the direct limit of the centers of such a direct limit is the center of the direct limit, when $\operatorname{tr}(I)=1$ in each approximating integration algebra, so that the ring of the direct limit is certainly a factor. In [109A] a variety of other constructions related to, although less simple than, the present one are given and shown to lead to the same factor. 
Further concrete examples of noncommutative integration algebras occur notably in the theory of harmonic analysis on locally compact groups, in ergodic theory, and in the theory of the Fermi-Dirac quantum field. It is not difficult in practice to determine whether a given system is an integration algebra, and a substantial complex of useful results otherwise obtainable only with considerable difficulty, and, in some cases, not at all, then follows ([88] and, slightly later, in [36] regarding the elementary aspects). The rings $L$ and $R$ generated, respectively, by the left and right multiplications by the elements of an integration algebra, acting on the corresponding $L_{2}$ space, say $\boldsymbol{H}$, obviously commute; what is less obvious, though relevant in several connections in harmonic analysis (a rather key point, e.g., in [57]), is that each exhausts the commutor of the other, i.e., consists of all bounded linear operators commuting with the other. The canonical adjunction on the integration algebra $a \rightarrow a^{*}$ induces a conjugation on $H$ which obviously carries $L$ into $R$ but, less obviously, leaves fixed the self-adjoint elements of the common center $L \cap R$. Conversely, if $\boldsymbol{S}$ is any ring of operators with a gage $m$ and if $J$ is a conjugation such that $J \boldsymbol{S} J=\boldsymbol{S}^{\prime}$, where $\boldsymbol{S}^{\prime}$ denotes the commutor of $\boldsymbol{S}$, with $J T J=T^{*}$ for $T \in S \cap S^{\prime}$, then the system $(S, m, J)$ is essentially a system of the type just indicated, with $S=L$, obtainable from an integration algebra. Such special systems or 'standard spaces' $(S, m, J)$ play much the same role in the structure theory of general rings of operators on Hilbert space as do conventional measure spaces in the case of commutative rings, or, more precisely, the system $(S, m, J)$ associated with the measure space, where $S$ is the multiplication ring of the space, $m$ assigns to any projection in $S$ the measure of the corresponding set, and $J$ is ordinary complex conjugation. Any two (nonsingular) gages are mutually absolutely continuous, as in the conventional situation (for the so-called 'localizable spaces' [93] which are relevant in spectral theory), and similarly the corresponding left-multiplication algebras, consisting of the left multiplications by elements of $S$ acting on $L_{2}(S, m)$, are unitarily equivalent. Any ring on which a nonsingular gage is defined-i.e., ring with no so-called type III component, in a representation of the ring as a direct sum of two rings-is algebraically isomorphic with its left multiplication ring formed in this way. This means that it is, within 'multiplicity,' a standard ring, obtained by removal of the specific gage from the standard space structure, just as any abelian ring is, apart from multiplicity, a maximal abelian ring; and just as the latter is unique within unitary equivalence, so is the associated standard ring in the general case. In the case of type III rings, multiplicity 
questions are essentially trivial (see [38]), so that in this way the problem of the structure of general rings of operators may be reduced to the corresponding problem for standard rings together with the analysis of the multiplicity, which proceeds also in a fashion analogous to that previously established in the abelian case.

The calculus of measurable operators [88] also makes possible the effective treatment of certain unbounded operators which occur in specific analytical connections. Any vector $x$ in the Hilbert space $\boldsymbol{H}=L_{2}(\boldsymbol{A}, E)$ associated with an integration algebra determines operators of left and right multiplication, definable as the closures of the applications of the operators to the image of $A$ in $H$, definable, in turn, by approximation with the multiplication given in $\boldsymbol{A}$. A special case of this is, for example, the operation of convolution by a squareintegrable function $f$ on a locally compact unimodular group $G$, acting on $L_{2}(G)$. If $f$ is integrable it is easily seen that the adjoint of this operation is convolution by $f^{*}$, where $f^{*}(x)=\bar{f}\left(x^{-1}\right)$, but it is not easy to deduce this for general elements of $L_{2}(G) .{ }^{9}$ This follows immediately from the measurability of all such operators, relative to the standard ring associated with $(A, E)$. This measurability also implies the much deeper result, apparently unobtainable in any direct fashion, that all such operators form a *algebra relative to the usual algebraic operations for partially defined operators followed by the operation of closure. In particular, any formally hermitian polynomial in these convolution operators is essentially self-adjoint on any of a wide class of domains; on the other hand, purely from the standpoint of general harmonic analysis, it is far from clear that the domain of such a polynomial has any nonzero vector.

Concepts similar to that of the integration algebra have developed from the theory of rings of operators, harmonic analysis on groups, and measure and ergodic theory. The work of von Neumann and Murray is frequently suggestive of the introduction of algebraicintegration-theoretic concepts, and in [67] they write of their results: 'This leads to an interesting and entirely new type of infinite hypercomplex system, which is at the same time a Hilbert space. A subsequent paper will be devoted to their independent study.' However, the indicated article did not appear. The abstract by Rokhlin [77A] introduced a system termed a 'unitary ring,' based on a Hilbert space some of whose elements were unbounded as multiplication operators. The ' $H$-system' introduced by Ambrose in [1] for the treat-

- Cf., e.g., the direct proof of the self-adjointness of the operation of convolution by a self-adjoint element of $L_{2}(G)$ which is given in [86]. 
ment of the $L_{2}$-convolution algebra of a unimodular locally compact group differed from the unitary ring primarily in its non-abelian character. The axioms for an $H$-system are difficult to verify in practice and the proof offered in [1] that $L_{2}(G)$ is an $H$-system is in error. In [88], the term 'Hilbert algebra' was introduced for a concept equivalent to that of the integration algebra on which the present exposition is based. The general theory of such algebras combined with the obvious Hilbert algebra character associated with $L_{2}(G)$ showed that it was indeed an $H$-system also, but the implications of this by virtue of [1] were subsumable under the elementary theory of Hilbert algebras on which the deduction was based.

In [37] Godement introduced shortly afterwards the notion of 'algèbre unitaire' which was likewise equivalent to that of integration algebra. The elementary theory was developed, along lines basically similar to those of the corresponding section in [88], and applied to the correlation of the notion with that of $H$-system in [73]. The cases of nonunimodular groups and type III rings, which do not provide integration algebras, suggested the generalization by Dixmier [17A] to 'algèbres quasi-unitaires.' They bear a nontrivial integration-theoretic structure only to the extent that the 'quasi' may be deleted, so that, while useful for algebraic purposes, they are logically excludable from the present exposition.

The theory of rings of operators in relation to conventional integration theory was thus far from being the only stimulus to the development of noncommutative integration theory. Some basic results in the theory of integration algebras were first proved in the context of harmonic analysis on groups. For example, the commutor theorem, to the effect that any operator which commutes with all right multiplications in an integration algebra is in the ring generated by the left multiplications, was first proved in a form in which it asserted that for any unimodular group $G$, any operator which commutes with any right translation is in the ring generated by the left translations [85]. The proof of the general integration algebra theorem ([88]; the proof in [36] is essentially the same) is similar to the central part of the proof of the cited group-theoretic result. The most important example, as well as the most significantly integration-theoretic, is that of the problem of the development of an effective common extension to the Plancherel theorem for abelian groups and the Peter-Weyl theorem for compact ones. This had been under consideration for some time (cf. [84]) before the appearance of von Neumann's paper [112] on direct integral theory supplied the crucial theory for making the connection with the general theory of rings of 
operators. ${ }^{10}$ This paper showed that a gage, or 'weight' function, in von Neumann's terminology, could be decomposed into a direct integral of dimension functions into which the given ring decomposed relative to its center. Much consideration had previously been given to the linear functional $E\left(L_{f}\right)=f(e)$, where $L_{f}$ denotes left convolution by the smooth function $f$ on $L_{2}(G), G$ being the given group, which had been shown to have a number of properties qualitatively describable as those of a noncommutative integral. The connection came about through the reformulation and extension of $E$ as follows. Let $P$ be any projection in the ring of operators generated by left translation; then if $P$ has the form $L_{f}$ for some element $f \in L_{2}(G)$, $m(P)$ is defined as $\|f\|^{2}$; if $P$ does not have this form, then $m(P)$ is defined as $+\infty$. (In a formal way, $m(P)$ may be identified with $E(P)$.) It could then be shown that $m$ was a nonsingular gage and the von Neumann reduction theory for weights brought to bear.

In the meantime, Gelfand and Nalmark in their highly germinal paper [29] had formulated an analogue to the Plancherel theorem in the case of the Lorentz group, proceeding along quite different and relatively classical lines. In later work [30], [31] this result was extended by them to the complex classical groups and, in part, to the real groups. Their formulas were of a highly explicit character which did not, however, make fully clear the connection with the general theory of semi-simple Lie algebras, as did the later work of HarishChandra [43] applicable to all the semi-simple complex groups. Probably the deepest successes of the extensive recent development of the theory of infinite-dimensional representations of Lie groups lie in the development of these formulas. It should be realized, however, that once a classification of the irreducible unitary representations of a semi-simple Lie group $G$ has been made, the derivation of a 'Plancherel formula' for the group consists precisely in the computation of a certain measure on the subsets of the parameter-space in terms of which the representations are labelled. The existence and basic properties of this measure are known from the general Plancherel theorem, in which formulation it is also known to be unique.

To describe this result, it should be recalled that the von Neumann

${ }^{10}$ The existence of this work, whose publication was delayed for a decade, had been indicated in earlier published work, but without any indication of the primarily relevant part dealing with weight functions. Von Neumann was aware of the interest in extending Plancherel's theorem, but expressed doubt as to its feasibility on the grounds that the real unimodular group in two dimensions had no unitary representations into finite factors. 
theory showed that the system $(L, m)$, where $L$ is the ring generated by the left translations, was a direct integral-symbolically,

$$
(L, m)=\int\left(L_{\lambda}, m_{\lambda}\right) d \mu(\lambda),
$$

where $\left(L_{\lambda}, m_{\lambda}\right)$ is a factorial ring-cum-gage, and $\lambda$ a real parameter. From this it is deducible ([86];11 see also below) that if $f$ is a function in $L_{1}(G) \cap L_{2}(G)$, then

$$
\int_{G}|f(x)|^{2} d x=\int \operatorname{tr}_{\lambda}\left(F_{\lambda}^{*} F_{\lambda}\right) d \mu(\lambda),
$$

where $F$ denotes the operator of left convolution by $f, F_{\lambda}$ the component of this operator in the constituent $L_{\lambda}$, and $\operatorname{tr}_{\lambda}$ the trace function corresponding to the gage $m_{\lambda}$. This is basically the content of the Plancherel formula except that the parameter $\lambda$ is not yet a label for a group representation. In a formal way, however, it determines a group representation, inasmuch as the formal map $F \rightarrow F_{\lambda}$ is a representation. This is readily done with the help of a later approach to reduction theory $([87, \mathrm{I}])$ which exploited the use of states and simple integration-theoretic ideas (cf. also the slightly later work [36] which is, in part, based on similar ideas). The use of real parameters $\lambda$ was eliminated in favor of one in any of a variety of spaces, which could in particular be chosen as perfect, in which case the highly intricate measurability problems involved in the von Neumann theory were greatly reduced. There is then for all $\lambda$ a corresponding continuous unitary representation of $G, R_{\lambda}$, and the formula then becomes virtually of the classical type with $F_{\lambda}$ having the form

$$
F_{\lambda}=\int_{G} R_{\lambda}(a) f(a) d a .
$$

11 In [62] essentially the same result is given, but only the simplest of the four lemmas providing the offered proof is correctly established. Among the difficulties are the following. Both in the proofs of Lemmas 7.1 and 7.4 (p. 551, lines 13-14 and p. 553, lines 9-10 from bottom) it is tacitly assumed as obvious that if $f$ is an element of $L_{2}(G)$ defining a bounded convolution operator, then the same is true of $f^{*}$. This follows from [88], but no independent proof was offered in the reply to our communication pointing out this deficiency in [62]. In the proof of Lemma 7.3 it is similarly assumed that if $f$ is an element of $L_{2}$, convolution by which agrees on $L_{1} \cap L_{2}$ with a bounded operator on $L_{2}$, then the convolution operator as defined by the usual integral is itself bounded. The interested reader may compare the submission dates of [62] and [86] and note that the otherwise exhaustive introduction to [62] contains no mention of the material in it just described, which is placed at the end of the paper and has no organic connection with its earlier parts. 
It may also be extended to arbitrary functions in $L_{2}(G)$, as in the case of the classical Plancherel theorem. The integral over the parameter space may be formulated as an integral over the space $P$ of all continuous irreducible representations of $G$ by forming the measuretheoretic quotient of the parameter space modulo the equivalence relation of corresponding to unitarily equivalent representations. It then has the form, identical with that usually employed in connection with semi-simple Lie groups, although for slightly more general functions $f$,

$$
\int_{G}|f(x)|^{2} d x=\int_{P} \operatorname{tr}_{R}\left(F_{R}^{*} F_{R}\right) d \mu(R), \text { where } \quad F_{R}=\int_{G} R(a) f(a) d a .
$$

Moreover, the measure $\mu$ is essentially unique.

What is involved in the derivation of explicit such formulas for particular groups is thus, basically, computation of the measure $\mu$ in terms of the parameters in terms of which the representations have been labelled. In principle, there is no uniqueness to these labelsother than the trivial label given by the identity map-so far as is presently known, nor is there any uniqueness to the corresponding reference measure in the parameter-space, in terms of which the Plancherel measure is usually given by its density function. There is naturally a corresponding ambiguity in the Plancherel measure, but it is of the same type as that discussed in the previous section, involving only a transference from one space to another of the measure.

From the viewpoint of general understanding rather than explicit computation the Plancherel measure may be best regarded as a gage on the center $\boldsymbol{C}$ of the ring of operators $L$ generated by left (or, equivalently, of right) translations. Any set $S$ of factorial unitary representations determines a projection in $\mathbf{C}$-namely that on the subspace of all elements of $L_{2}(G)$ which transform in accordance with a direct integral of elements of the representations-and the Plancherel measure of $S$ is the gage of the corresponding projection. ${ }^{12}$ The complete Boolean algebra of these projections is naturally representable, for separable groups, as a Boolean algebra of sets of factorial representations, on which a measure is defined in this way. There is, in general, an ambiguity in the normalization of the trace functions $\operatorname{tr}_{R}$, but there is a natural normalization except in the case in which

12 When $G$ is abelian these projections are in one-to-one correspondence with the measurable sets modulo null sets in the dual group $G^{*}$, and the Plancherel measure is the Haar measure; in [79], Haar measure on $G^{*}$ is constructed in essentially this fashion. Thus $\mu$ is in a sense dual to $m$, which is the origin of the term "dual gage" used for $\mu$ in [86]. 
factorial components of type $\mathrm{II}_{\infty}$ are present, which is rare (in fact, a set of type $\mathrm{II}_{\infty}$ of positive measure has not yet been observed for any group, though there is no special reason to doubt that Lie groups will eventually be found for which they exist). In particular, in the cases of abelian, compact, discrete, and semi-simple Lie groups, such components cannot arise. For the especially interesting, though analytically most complicated, case of a semi-simple Lie group or any other so-called 'type I' group, the Plancherel measure $\mu$, with the standard normalization of the trace functions, is readily given directly in terms of the gage $m$, e.g., in the following fashion. A projection $P$ in the ring $L$ may be called relatively minimal in case it has the property that if $Q$ is any other projection in $L$ such that $Q \leqq P$, then necessarily $Q=P K$ with $K$ in $C$; and relatively nonsingular if $P K=0$ with $K$ in $C$ only when $K=0$. Projections with both of these properties exist, on the basis of the general theory of type I rings ([87, II]; also in [18]), and if $P_{0}$ is any one of them, the Plancherel measure is given by the formula

$$
\mu(E)=m\left(E P_{0}\right), \quad E \in C,
$$

as is also clear from the general theory. On the other hand, an explicit determination of an operator $P_{0}$, which is highly nonunique although the corresponding measure is unique, is not easy. In the nature of the situation, it is not to be expected that there exists any explicit expression for the Plancherel measure which is applicable to broad classes of groups, but a conjecture emerging from the work of Gelfand and his associates, of a conceptually quite simple if analytically implicit character, is given in the paper of Kirillov [49]; its applicability to nilpotent groups is established there, by methods quite distinct from those involved in establishing it in the semisimple case treated earlier by Gelfand and collaborators, and it may quite conceivably be valid for arbitrary connected Lie groups, or at least those of type I.

A completely different concrete analytical situation in which noncommutative integration theory naturally arises, the theory of FermiDirac quantization, is described in [91], [94], and [100]. The approximately finite factor of type $\mathrm{II}_{1}$ arises here in a natural way from a weak distribution on Hilbert space, the Clifford distribution, which is noncommutative but nevertheless has a role closely analogous to that of the isonormal distribution, from which, in a certain sense, it differs only in sign. This approach to the Fermi-Dirac quantum field makes possible a more general and explicitly invariant treatment of it than has yet been attained by other methods; it seems well suited to the rigorous treatment of the Dirac hole theory, 
which has not yet been given in the literature; it is planned that this will be detailed elsewhere.

\section{BIBLIOGRAPHY}

1. W. Ambrose, The $L_{2}$-system of a unimodular group. I, Trans. Amer. Math. Soc. 65 (1949), 27-48.

2. R. J. Blattner, Automorphic group representations, Pacific J. Math. 8 (1958), 665-677.

2A. S. Bochner, Monotone funktionen, Stieltjessche Integrale und harmonische analyse, Math. Ann. 108 (1933), 378-410.

3. S. Bochner, Stochastic processes, Ann. of Math. (2) 48 (1947), 1014-1061.

4. - Harmonic analysis and the theory of probability, Univ. California Press, Berkeley, Calif., 1955.

4A. N. Bourbaki, Elements de mathematique. XIII, Hermann, Paris, 1952.

5. R. H. Cameron, The translation pathology of Wiener space, Duke Math. J. 21 (1954), 623-627.

6. R. H. Cameron and W. T. Martin, Transformations of Wiener integrals under translations, Ann. of Math. (2) 45 (1944), 386-396.

7. - An expression for the solution of a class of non-linear integral equations, Amer. J. Math. 66 (1944), 281-298.

8. - Transformations of Wiener integrals under a general class of linear transformations, Trans. Amer. Math. Soc. 58 (1945), 184-219.

9. - Fourier-Wiener transforms of functionals belonging to $L_{2}$ over the space $C$, Duke Math. J. 14 (1947), 99-107.

10. - The behavior of measure and measurability under change of scale in Wiener space, Bull. Amer. Math. Soc. 53 (1947), 130-137.

11. - The transformation of Wiener integrals by non-linear transformations, Trans. Amer. Math. Soc. 66 (1949), 253-283.

12. C. Carathéodory, Mass und Integral und ihre Algebraisierung, Birkhäuser Verlag, Basel and Stuttgart, 1956.

12A. J. M. Cook, The mathematics of second quantization, Trans. Amer. Math. Soc. 74 (1957), 222-245.

13. P. J. Daniell, A general form of integral, Ann. of Math. (2) $19(1917 / 18)$, 279-294.

14. P. A. M. Dirac, The principles of quantum mechanics, 3rd ed., Oxford Univ. Press, Oxford, 1947.

15. J. Dixmier, Les fonctionnelles linéaires sur l'ensemble des operateurs bornés d'un espace de Hilbert, Ann. of Math. (2) 51 (1950), 387-408.

16. - Sur certaines espaces consideres par M. H. Stone, Summa Brasil. Math. 2 (1951), 151-182.

17. - Formes lineaires sur un anneau d'operateurs, Bull. Soc. Math. France 81 (1953), 9-39. 322.

17A. - Algebres quasi-unitaires, Comment. Math. Helv. 26 (1952), 275-

18. - Les algèbres d'operateurs dans l'espace hilbertien, Gauthier-Villars, Paris, 1957

19. H. A. Dye, The Radon-Nikodym theorem for finite rings of operators, Trans. Amer. Math. Soc. 72 (1952), 243-280.

20. J. Feldman, On the Schrödinger and heat equations for bad potentials, Trans. Amer. Math. Soc. 108 (1963), 251-264. 
21. R. P. Feynman, Space-time approach to non-relativistic quantum mechanics, Rev. Modern Phys. 20 (1948), 367-387.

22. - An operator calculus having applications in quantum electrodynamics, Phys. Rev. (2) 84 (1951), 108-128.

23. K. O. Friedrichs, Mathematical aspects of the quantum theory of fields, Interscience, New York, 1953.

24. K. O. Friedrichs and H. N. Shapiro, Integration over Hilbert space and outer extensions, Proc. Nat. Acad. Sci. U.S.A. 43 (1957), 336-338.

25. K. O. Friedrichs and colleagues, Seminar on Integration of Functionals, Mimeographed notes published by New York University, Institute of Mathematical Sciences, 1957.

26. H. Furstenberg, Stationary processes and prediction theory, Princeton Univ. Press, Princeton, N. J., 1960.

27. I. M. Gelfand, Normierte Ringe, Mat. Sb. (N.S.) 9 (51) (1941), 3-24.

28. —- Generalized random processes, Dokl. Akad. Nauk SSSR 100 (1955), 853-856. (Russian)

29. I. M. Gelfand and M. A. NaImark, Unitary representations of the Lorentz group, Izv. Akad. Nauk SSSR Ser. Mat. 11 (1947), 411-504. (Russian)

30. - Unitary representations of the classical groups, Trudy Mat. Inst. Steklov., Vol. 36, Izdat. Akad. Nauk SSSR, Moscow, 1950. (Russian)

31. I. M. Gelfand and M. I. Graev, Analogue of the Plancherel formula for the classical groups, Trudy Moskov. Mat. Obšc. 4 (1955), 375-404 (Russian); Amer. Math. Soc. Transl. (2) 9 (1958), 123-154.

32. I. M. Gelfand, D. A. Rykov, and G. E. Shilov, Commutative normed rings, Uspehi Mat. Nauk 1 (1946), 48-146 (Russian); Amer. Math. Soc. Transl. (2) 5 (1957), 115-220.

33. I. M. Gelfand and N. Y. Vilenkin, Generalized functions, Vol. 4, Moscow, 1961.

34. I. M. Gelfand and A. M. Yaglom, Integration in functional spaces and its applications in quantum physics, J. Mathematical Phys. 1 (1960), 48-69.

35. A. M. Gleason, Measures on the closed subspaces of a Hilbert space, J. Math. Mech. 6 (1957), 885-893.

36. R. Godement, Sur la theorie des représentations unitaires, Ann. of Math. (2) 53 (1951), 68-124.

37. —- Thêrie des caractères. I. Algèbres unitaires, Ann. of Math. (2) 59 (1954), 47-62.

38. E. L. Griffin, Jr., Some contributions to the theory of rings of operators, Trans. Amer. Math. Soc. 75 (1953), 471-504; II, ibid. 79 (1955), 389-400.

39. L. Gross, Integration and non-linear transformations in Hilbert space, Trans. Amer. Math. Soc. 94 (1960), 404-440.

40. - Measurable functions on Hilbert space, Trans. Amer. Math. Soc. 105 (1962), 372-390.

41. - - Harmonic analysis on Hilbert space, Mem. Amer. Math. Soc. No. 46 (1963).

42. - Classical analysis on a Hilbert space, Proc. Conf. Analysis in Function Space, Cambridge, Mass., 1964, pp. 51-68.

43. Harish-Chandra, The Plancherel formula for complex semisimple Lie groups, Trans. Amer. Math. Soc. 76 (1954), 485-528.

44. K. Ito, Stationary random distributions, Mem. Coll. Sci. Univ. Kyoto 28 (1953), 209-223.

45. M. Kac, Probability and related topics in physical science, Proc. Summer Seminar, Boulder, Colo., 1957, Vol. I, Interscience, New York, 1959.

46. R. V. Kadison, On the additivity of the trace in finite factors, Proc. Nat. Acad Sci. U.S.A. 41 (1955), 385-387. 
47. S. Kakutani, Concrete representation of abstract $(L)$-spaces and the mean ergodic theorem, Ann. of Math. (2) 42 (1941), 523-537.

48. - Determination of the spectrum of the flow of Brownian motion, Proc. Nat. Acad. Sci. U.S.A. 36 (1950), 319-323.

48A. - Spectral analysis of stationary Gaussian processes, Proc. 4th Berkeley Sympos. Probability, Vol. 2, 1961; pp. 239-247.

49. A. Kirillov, Unitary representations of nilpotent Lie groups, Russian Math. Surveys 17 (1962), 53-104.

50. A. Kolmogoroff, Grundbegriffe der Wahrscheinlichkeitsrechnung, Ergebnisse der Mathematik und ihrer Grenzgebiete, Springer, Berlin, 1933; pp. 1-62.

51. R. A. Kunze, $L_{p}$-Fourier transforms on locally compact unimodular groups, Trans. Amer. Math. Soc. 89 (1958), 519-540.

52. C. Loewner, Grundzïge einer Inhaltlehre im Hilbertschen Raume, Ann. of Math. (2) 40 (1939), 816-833.

53. L. H. Loomis, On the representation of $\sigma$-complete Boolean algebras, Bull. Amer. Math. Soc. 63 (1947), 757-760.

54. - Note on a theorem of Mackey, Duke Math. J. 19 (1952), 641-645.

55. G. W. Mackey, $A$ theorem of Stone and von Neumann, Duke Math. J. 16 (1949), 313-326.

56. - Imprimitivity for representations of locally compact groups. I, Proc. Nat. Acad. Sci. U.S.A. 35 (1949), 537-545.

57. - Induced representations of locally compact groups. II. The Frobenius reciprocity theorem, Ann. of Math. (2) 58 (1953), 193-221.

58. —, Unitary representations of group extensions. I, Acta Math. 99 (1958), 265-371.

59. — Point realizations of transformation groups, Illinois J. Math. 6 (1962), $327-335$.

60. D. Maharam, Homogeneous measure algebras, Proc. Nat. Acad. Sci. U.S.A. 28 (1941), 108-111.

61. - An algebraic characterization of measure algebras, Ann. of Math. (2) 48 (1947), 154-167.

62. F. Mautner, Unitary representations of locally compact groups. II, Ann. of Math. (2) 52 (1950), 528-556.

63. - On eigenfunction expansions, Proc. Nat. Acad. Sci. U.S.A. 39 (1953), 49-53.

64. E. J. McShane, Remark concerning integration, Proc. Nat. Acad. Sci. U.S.A. 35 (1949), 46-49.

65. - Integrals devised for special purposes, Bull. Amer. Math. Soc. 69 (1963), 597-627.

65A. J. Milkman, Hermite polynomials, Hermite functionals and their integrals in real Hilbert space, Riv. Mat. Univ. Parma 6 (1955), 65-88.

66. F. J. Murray and J. von Neumann, On rings of operators, Ann. of Math. 37 (1936), 116-229.

67. - On rings of operators. II, Trans. Amer. Math. Soc. 41 (1937), 208-248.

68. - On rings of operators. IV, Ann. of Math. (2) 44 (1943), 716-808.

69. M. Nakamura and H. Umegaki, On a proposition of von Neumann, Kōdai Math. Sem. Rep. 8 (1956), 142-144.

70. H. Nakano, Hilbert algebras, Tôhoku Math. J. 2 (1950), 4-23.

71. Edward Nelson, Feynman integrals and the Schrödinger equation, J. Mathematical Phys. 5 (1964), 332-343.

72. R. E. A. C. Paley and N. Wiener, Fourier transforms in the complex domain, Amer. Math. Soc. Colloq. Publ. Vol. 19, Amer. Math. Soc., Providence, R. I., 1934. 
73. R. Pallu de la Barrière, Algebres unitaires et espaces d'Ambrose, Ann. Sci. Ecole Norm. Sup. (3) 70 (1953), 381-401.

73A. - Sur les algëbres d'operateurs dans les espaces hilbertiens, Bull. Soc. Math. France 82 (1954), 1-52.

74. M. Plancherel, Contribution à l'étude de la representation d'unefonction arbitraire par des integrals défnie, Rend. Circ. Mat. Palermo 30 (1910), 289-335.

75. A. Plessner, Eine Kennzeichnung der total-stetigen Funktionen, J. Reine Angew. Math. 160 (1929), 26-32.

76. Y. V. Prohorov, The method of characteristic functionals, Proc. 4th Berkeley Sympos. Probability, Vol. 2, 1961; pp. 403-420.

77. L. Pukanszky, The theorem of Radon-Nikodym in operator-rings, Acta Sci. Math. (Szeged) 15 (1954), 149-156. 646.

77A. V. A. Rokhlin, Unitary rings, Dokl. Akad. Nauk SSSR 59 (1948), 643-

78. Proceedings of the Symposium on Time Series Analysis, M. Rosenblatt, editor, pp. 279-348, Wiley, New York, 1963.

79. D. A. Rykov, Harmonic analysis on commutative groups, Trudy Mat. Inst. Steklov. 14 (1945).

80. S. Saks, Theory of the integral, 2nd ed.; Note II by S. Banach, Lebesgue integration in abstract spaces, pp. 320-330.

81. V. Sazonov, On characteristic functionals, Teor. Verojatnost. i Primenen. 3 (1958), 201-205.

82. R. Schatten, The space of completely continuous operators on a Hilbert space, Math. Ann. 134 (1957), 47-49.

83. I. Segal, Postulates for general quantum mechanics, Ann. of Math. (2) 48 (1947), 930-948.

84. - $-A$ kind of abstract integration pertinent to locally compact groups. I, Abstract, Bull. Amer. Math. Soc. 55 (1949), 46.

85. - The two-sided regular representation of a unimodular locally compact group, Ann. of Math. (2) 51 (1950), 293-298.

86. - An extension of Plancherel's formula to separable unimodular groups, Ann. of Math. (2) 52 (1950), 272-292.

87. - Decompositions of operator algebras. I, II. Multiplicity theory, Mem. Amer. Math. Soc. No. 9, (1951).

88. - A non-commutative extension of abstract integration, Ann. of Math. (2) 57 (1953), 401-457.

89. - Abstract probability spaces and a theorem of Kolmogoroff, Amer. J. Math. 76 (1954), 721-732.

90. — Tensor algebras over Hilbert spaces, Trans. Amer. Math. Soc. 81 (1956), $106-134$.

91. - Tensor algebras over Hilbert spaces. II, Ann. of Math. (2) 63 (1956), $160-175$.

92. - Ergodic subgroups of the orthogonal group on a real Hilbert space, Ann. of Math. (2) 66 (1957), 297-303.

93. —_ Equivalences of measure spaces, Amer. J. Math. 73 (1951), 275-313.

94. - Distributions in Hilbert space and canonical systems of operators, Trans. Amer. Math. Soc. 88 (1958), 12-41.

95. - A theorem on the measurability of group-invariant operators, Duke Math. J. 26 (1959), 549-552.

95A. - Foundations of the theory of dynamical systems of infinitely many degrees of freedom. I, Mat. Fys. Medd. Danske Vid. Selsk. 31 (1959), no. 12. 
96. - Transforms for operators and symplectic automorphisms over a locally compact abelian group, Math. Scand. 13 (1963), 31-43.

97. - Mathematical problems of relativistic physics, Lectures in Applied Mathematics, Amer. Math. Soc., Providence, R. I., 1963.

98. T. I. Seidman, Linear transformations of a functional integral, Comm. Pure Appl. Math. 12 (1959), 611-621; correction, ibid. 16 (1963), 95-96; II, ibid. 17 (1964), 493-508.

99. D. Shale, Linear symmetries of free boson fields, Trans. Amer. Math. Soc. 103 (1962), 149-167.

100. D. Shale and W. F. Stinespring, States of the Clifford algebra, Ann. of Math. (2) 80 (1964), 365-381.

101. W. F. Stinespring, Integration theorems for gages and duality for unimodular groups, Trans. Amer. Math. Soc. 90 (1959), 15-56.

102. M. H. Stone, Linear transformations in Hilbert space, Amer. Math. Soc. Colloq. Publ. Vol. 15, Amer. Math. Soc., Providence, R. I., 1932.

102A. - On one-parameter unitary groups in Hilbert space, Ann. of Math. 33 (1932), 643-648.

103. - The theory of representations of Boolean algebras, Trans. Amer. Math. Soc. 40 (1936), 37-111.

104. - Applications of the theory of Boolean rings to general topology, Trans. Amer. Math. Soc. 41 (1937), 375-481.

105. - Notes on integration. I, Proc. Nat. Acad. Sci. U.S.A. 34 (1948), 336-342; II, ibid. 34 (1948), 447-455; III, ibid. 34 (1948), 483-490; IV, ibid. 35 (1949), 50-58.

106. - A general theory of spectra. I, Proc. Nat. Acad. Sci. U.S.A. 26 (1940), 280-283; II, ibid. 27 (1941), 83-87.

107. J. von Neumann, Die Eindeutigkeit der Schrödingerschen Operatoren, Math. Ann. 104 (1941), 570-578.

108. —, Über Funktionen von Funktionaloperatoren, Ann. of Math. 32 (1931), 191-226. 1932.

109. - Mathematische Grundlagen der Quantenmechanik, Springer, Berlin,

109A. - Approximative properties of matrices of high order, Portugal. Math. 3 (1942), 1-62.

110. - On an algebraical generalization of the quantum mechanical formalism. I, Mat. Sb. (N.S.) 1 (437) (1936), 415-484.

111. - On rings of operators. III, Ann. of Math. 41 (1940), 94-161.

112. - On rings of operators. Reduction theory, Ann. of Math. (2) 50 (1949), 401-485.

113. A. Weil, L'intégration dans les groupes topologiques et ses applications, Hermann, Paris, 1938.

114. - Sur certains groupes d'operateurs unitaires, Acta Math. 111 (1964), 247-302.

115. N. Wiener, Differential space, J. Math. Phys. M.I.T. 2 (1923), 131-174.

116. —, The homogeneous chaos, Amer. J. Math. 60 (1938), 896-936.

117. - The Fourier integral and certain of its applications, Cambridge Univ. Press, Cambridge, 1933.

Massachusetts Institute of Technology and University of Aarhus, Denmark 